\title{
How the power spectrum of dust continuum images may hide the presence of a characteristic filament width
}

\author{
A. Roy ${ }^{1,2}$, Ph. André ${ }^{1}$, D. Arzoumanian ${ }^{3}$, M.-A. Miville-Deschênes ${ }^{1,4}$, V. Könyves ${ }^{1}$, N. Schneider ${ }^{5}$, \\ S. Pezzuto ${ }^{6}$, P. Palmeirim ${ }^{7}$, and J. M. Kirk $^{8}$ \\ ${ }^{1}$ Laboratoire d'Astrophysique (AIM), CEA, CNRS, Université Paris-Saclay, Université Paris Diderot, Sorbonne Paris Cité, \\ 91191 Gif-sur-Yvette, France \\ e-mail: aroy@cita.utoronto.ca, pandre@cea.fr \\ 2 Laboratoire d'Astrophysique de Bordeaux, Univ. Bordeaux, CNRS, B18N, Allée G. Saint-Hilaire, 33615 Pessac, France \\ 3 Department of Physics, Nagoya University, Furo-cho, Chikusa-ku, Nagoya, Aichi 464-8602, Japan \\ ${ }^{4}$ Institut d'Astrophysique Spatiale, CNRS, Univ. Paris-Sud, Université Paris-Saclay, Bâtiment 121, 91405 Orsay Cedex, France \\ 5 I. Physik. Institut, University of Cologne, Zülpicher Str. 77, 50937 Koeln, Germany \\ 6 INAF - Istituto di Astrofisica e Planetologia Spaziali, Via Fosso del Cavaliere 100, 00133 Roma, Italy \\ 7 Instituto de Astrofísica e Ciências do Espaço, Universidade do Porto, CAUP, Rua das Estrelas, 4150-762 Porto, Portugal \\ 8 University of Central Lancashire, Preston, Lancashire PR1 2HE, UK
}

Received 21 February 2018 / Accepted 26 March 2019

\begin{abstract}
Context. Herschel observations of interstellar clouds support a paradigm for star formation in which molecular filaments play a central role. One of the foundations of this paradigm is the finding, based on detailed studies of the transverse column density profiles observed with Herschel, that nearby molecular filaments share a common inner width of $\sim 0.1 \mathrm{pc}$. The existence of a characteristic filament width has been recently questioned, however, on the grounds that it seems inconsistent with the scale-free nature of the power spectrum of interstellar cloud images.

Aims. In an effort to clarify the origin of this apparent discrepancy, we examined the power spectra of the Herschel/SPIRE $250 \mu \mathrm{m}$ images of the Polaris, Aquila, and Taurus-L1495 clouds in detail and performed a number of simple numerical experiments by injecting synthetic filaments in both the Herschel images and synthetic background images.

Methods. We constructed several populations of synthetic filaments of $0.1 \mathrm{pc}$ width with realistic area filling factors $\left(A_{\text {fil }}\right)$ and distributions of column density contrasts $\left(\delta_{\mathrm{c}}\right)$. After adding synthetic filaments to the original Herschel images, we recomputed the image power spectra and compared the results with the original, essentially scale-free power spectra. We used the $\chi_{\text {variance }}^{2}$ of the residuals between the best power-law fit and the output power spectrum in each simulation as a diagnostic of the presence (or absence) of a significant departure from a scale-free power spectrum.

Results. We find that $\chi_{\text {variance }}^{2}$ depends primarily on the combined parameter $\delta_{\mathrm{c}}^{2} A_{\mathrm{fil}}$. According to our numerical experiments, a significant departure from a scale-free behavior and thus the presence of a characteristic filament width become detectable in the power spectrum when $\delta_{\mathrm{c}}^{2} A_{\mathrm{fil}} \gtrsim 0.1$ for synthetic filaments with Gaussian profiles and $\delta_{\mathrm{c}}^{2} A_{\text {fil }} \gtrsim 0.4$ for synthetic filaments with Plummer-like density profiles. Analysis of the real Herschel $250 \mu \mathrm{m}$ data suggests that $\delta_{\mathrm{c}}^{2} A_{\mathrm{fil}}$ is $\sim 0.01$ in the case of the Polaris cloud and $\sim 0.016$ in the Aquila cloud, significantly below the fiducial detection limit of $\delta_{\mathrm{c}}^{2} A_{\mathrm{fil}} \sim 0.1$ in both cases. In both clouds, the observed filament contrasts and area filling factors are such that the filamentary structure contributes only $\sim 1 / 5$ of the power in the image power spectrum at angular frequencies where an effect of the characteristic filament width is expected.

Conclusions. We conclude that the essentially scale-free power spectra of Herschel images remain consistent with the existence of a characteristic filament width $\sim 0.1 \mathrm{pc}$ and do not invalidate the conclusions drawn from studies of the filament profiles.
\end{abstract}

Key words. local insterstellar matter - submillimeter: ISM - stars: low-mass - infrared: diffuse background

\section{Introduction}

Recent Herschel imaging observations of nearby molecular clouds, for example, those obtained as part of the Herschel Gould Belt Survey (HGBS; André et al. 2010), indicate that filamentary structures are characterized by a common inner width $W_{\text {fil }} \sim 0.1 \mathrm{pc}$, with only a factor of approximately two spread around this value, over a wide range of column densities (Arzoumanian et al. 2011, 2019; Koch \& Rosolowsky 2015). If confirmed, the existence of such a characteristic filament width has remarkable implications for the star formation process and is one of the bases of a proposed filamentary paradigm for solar-type star formation (André et al. 2014). In particular, it may set a critical column density threshold above which most stars form in filamentary molecular clouds. For filaments of $\sim 0.1 \mathrm{pc}$ width and a typical gas temperature of $10 \mathrm{~K}$, the critical mass per unit length $M_{\text {line,crit }}=2 c_{\mathrm{s}}^{2} / G \sim 16 M_{\odot} \mathrm{pc}^{-1}$ (cf. Inutsuka \& Miyama 1997) indeed translates to a critical column density $\Sigma_{\text {gas,crit }} \sim M_{\text {line,crit }} / W_{\text {fil }} \sim 160 M_{\odot} \mathrm{pc}^{-2}$, which is close to the background column density threshold above which prestellar cores are found with Herschel in nearby regions (e.g., Könyves et al. 2015; Marsh et al. 2016). Above this threshold, the star formation rate is observed to be directly proportional to the mass of dense molecular gas in both nearby clouds and external galaxies (e.g., Gao \& Solomon 2004; Heiderman et al. 2010; Lada et al. 2010; Shimajiri et al. 2017).

Arzoumanian et al. (2011) suggested the existence of a characteristic filament width after fitting simple Gaussian or 
Plummer-like model profiles to the transverse profiles observed with Herschel for a broad sample of nearby filaments (see also Arzoumanian et al. 2019). In the analysis of Arzoumanian et al., thermally supercritical filaments (with $M_{\text {line }}>2 c_{\mathrm{s}}^{2} / G$ ) tend to have Plummer-like density profiles with a flat inner region of radius $R_{\text {flat }}$ and a decreasing power-law wing $\rho \propto r^{-2}$ at larger radii. In contrast, low column density, thermally subcritical filaments (with $M_{\text {line }}<2 c_{\mathrm{s}}^{2} / G$ ) tend to be better described by Gaussian density profiles.

Why molecular filaments seem to share such a characteristic width is still a debated theoretical problem (e.g., Hennebelle \& André 2013; Fischera \& Martin 2012; Federrath 2016; Auddy et al. 2016). In order to ascertain whether the presence of this possibly universal filament scale is robust, the observational data also need to be investigated using various other means. In a recent paper, Panopoulou et al. (2017) tested the possibility of identifying a characteristic scale using a power spectrum analysis. In their study they argued that, had there been a characteristic filament width, its signature should have manifested itself in the power spectrum of Herschel images of nearby clouds, either as a kink or as a change in slope at an angular frequency corresponding to the characteristic scale.

In the present paper, we revisit the latter issue from an observer's standpoint, exploring the parameter space with realistic filament properties consistent with the observational data, in particular taking into account realistic distributions of filament contrasts and area filling factors. To this end, we selected two extreme regions imaged by the HGBS, namely the Polaris translucent cloud, mainly dominated by low density subcritical $\left(M_{\text {line }}<M_{\text {line,crit }}\right)$ filaments, and the Aquila complex, which contains a fair population of high column density supercritical $\left(M_{\text {line }}>M_{\text {line,crit }}\right)$ filaments. We also used the B211/B213 field in the Taurus cloud, which is dominated by a single, marginally supercritical filament.

The layout of the paper is as follows. In Sect. 2 we describe the construction of synthetic filament images and their power spectra. In Sect. 3, we develop a diagnostic for the detection of a characteristic filament width in a power spectrum plot. We also develop a diagnostic for the detection of a characteristic filament width in a power spectrum plot. In Sects. 4 and 5, we perform a power spectrum analysis of the Herschel images of the Polaris and Aquila clouds, respectively, and compare the results to those obtained on synthetic maps after adding simulated filaments. In Sect. 6, we compared power spectra of a subregion of Taurus molecular cloud encompassing the Taurus main filament to a synthetic filament with similar physical properties as B211/B213. In Sect. 7, we investigate the combined effect of filament column density contrast $\left(\delta_{\mathrm{c}}\right)$ and area filling factor $\left(A_{\mathrm{fil}}\right)$. Finally, we summarize our results in Sect. 8.

\section{Construction of synthetic filaments and their power spectra}

Figure 1 shows an example of a synthetic filament with a transverse Gaussian profile and a projected spatial inner width (FWHM) of $0.1 \mathrm{pc}$ at a distance of $140 \mathrm{pc}$. Mathematically, the 2D image of a filament with a Gaussian profile can be expressed as

$I_{\text {Cylinder }}(x, y)=C\left[\delta^{\mathrm{L}}(a x+b y+c) \times \Pi_{\mathrm{L}}\right] \star G_{\theta_{\text {fil }}}(x, y)$,

where, $C$ is the amplitude factor (related to the filament contrast) of the delta line function $\delta^{\mathrm{L}}(a x+b y+c), \Pi_{\mathrm{L}}$ is a rectangle function, and the $\star$ symbol denotes the convolution operator. The

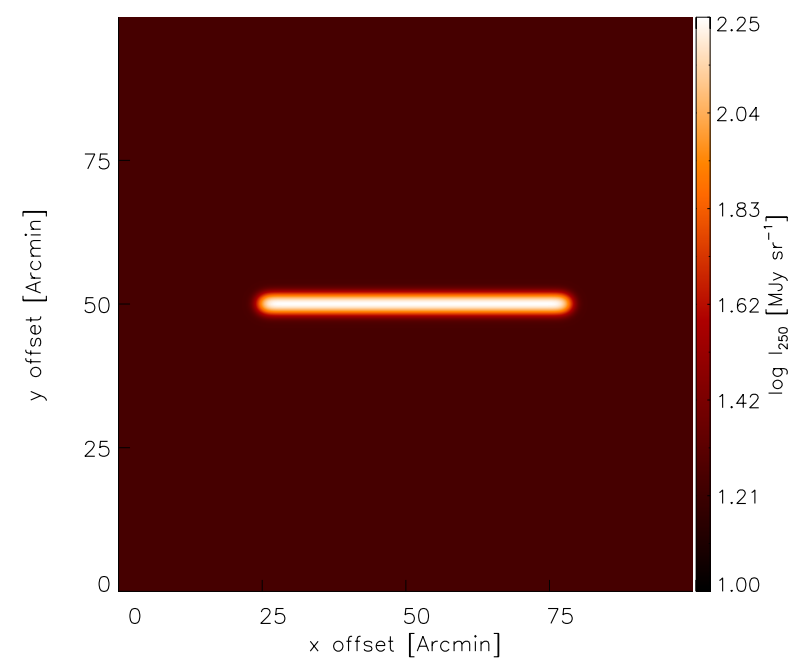

Fig. 1. Image of a simulated filament with a Gaussian transverse profile and a FWHM width of $0.1 \mathrm{pc}$, projected at a distance of $140 \mathrm{pc}$. Here, the level of filament contrast was adjusted so that $\delta_{\mathrm{c}} \sim 10$.

delta line function assumes a value of unity when its parameter $a x+b y+c=0$, and zero elsewhere. The expression $a x+b y+c=0$ is the equation of a straight line where the $a, b$ coefficients determine the slope of the straight line and $c$ is the intercept. The rectangle function, $\Pi_{\mathrm{L}}$, which has a value of unity over the length $L$ of the line function and zero elsewhere, transforms the line into a line-segment of length $L$. In order to make a Gaussian filament profile, we convolve the entire line segment $\left[\delta(a x+b y+c) \times \Pi_{\mathrm{L}}\right]$ with a Gaussian kernel, $G_{\theta_{\text {fil }}}(x, y)$, of full width at half maximum $F W H M=\theta_{\text {fil }}$.

The FWHM of the Gaussian kernel $=\theta_{\text {fil }}$ is chosen such that the projected spatial width is $W_{\text {fil }}$. To create a characteristic $W_{\text {fil }}$ inner width of a filament at a distance $d$, the required $\theta_{\text {fil }}$ is

$\theta_{\text {fil }} \simeq 147^{\prime \prime} \times\left(\frac{W_{\text {fil }}}{0.1 \mathrm{pc}}\right) \times\left(\frac{140 \mathrm{pc}}{d}\right)$

The choice of the parameter $C$ depends upon the required level of filament contrast defined as $\delta_{\mathrm{c}}=\left(I^{\text {peak }}-I^{\mathrm{bkg}}\right) / I^{\mathrm{bkg}}$ and on the dilution factor of the convolution kernel,

$C \approx \delta_{\mathrm{c}} \theta_{\text {fil }}$.

For example, in order to create a Gaussian filament profile of spatial $F W H M=0.1 \mathrm{pc}$ and contrast $\delta_{\mathrm{c}}=0.4$ at the distance of Polaris $(d=140$ pc, Falgarone et al. 1998), we used a Gaussian convolution kernel of $\theta_{\text {fil }} \sim 147^{\prime \prime}$ (see Eq. (3)), and a contrast amplitude of $C \sim 0.4 \times 147^{\prime \prime} / \theta_{\text {pix }}$, where $\theta_{\text {pix }}$ is the pixel size of the image. We adopted a pixel size $\theta_{\text {pix }}=6^{\prime \prime}$ for Herschel/SPIRE $250 \mu \mathrm{m}$ images (18.2" beam resolution).

The Fourier transform of a 2D image can be expressed as

$\hat{I}\left(k_{x}, k_{y}\right)=\int I(x, y) e^{-2 \pi i\left(k_{x} x+k_{y} y\right)} \mathrm{d} x \mathrm{~d} y$,

where $\mathrm{d} x \mathrm{~d} y$ is the infinitesimal surface area, and $\int \mathrm{d} x \mathrm{~d} y=S$ is the total surface area, $S$, covered by the map. For an image of a single cylindrical filament, most of the contribution to the integral in Eq. (4) comes from integration over the central part of the filament which encloses $75 \%$ of the total intensity fluctuations.

The power spectrum of a cylindrical intensity distribution can be written analytically as 


$$
\begin{aligned}
P_{\text {cylinder }}\left(k_{x}, k_{y}\right) & =\left|\hat{I}\left(k_{x}, k_{y}\right)\right|^{2}, \\
& =\mid F T\left(\left.\delta^{\mathrm{L}}(a x+b y+c)\left(k_{x}, k_{y}\right)\right|^{2} \hat{G}^{2}\left(k_{x}, k_{y}\right),\right. \\
& =\left|\hat{\delta}\left(b k_{x}-a k_{y}\right)\right|^{2} \hat{G}^{2}\left(k_{x}, k_{y}\right),
\end{aligned}
$$

where $\hat{\delta}\left(b k_{x}-a k_{y}\right)$ is the Fourier transform of the delta line function $\delta^{\mathrm{L}}(a x+b y+c)$ and $\hat{G}\left(k_{x}, k_{y}\right)$ is the Fourier transform of the convolution kernel. The power spectrum of the Gaussian kernel, $\hat{G}^{2}\left(k_{x}, k_{y}\right)$, is also a Gaussian, and its FWHM width $\Gamma_{\text {fil }}$ is related to the FWHM width $\theta_{\text {fil }}$ of $G_{\theta_{\text {fil }}}(x, y)$ through the relation:

$$
\begin{aligned}
\Gamma_{\text {fil }} & =\sqrt{8} \ln 2 / \pi \theta_{\text {fil }} \\
& \sim 0.6 / \theta_{\text {fil }} .
\end{aligned}
$$

One may thus expect a characteristic filament width $\theta_{\text {fil }}$ to lead to a signature in the power spectrum at angular frequencies $k_{\text {fil }} \sim \Gamma_{\text {fil }}$.

For multiple, randomly distributed filaments, as in the simulations discussed below, it is not possible to obtain the power spectrum analytically. We therefore used the IDL-based routine FFT to compute the power spectrum. Nevertheless, Eq. (5) is useful to appreciate how the power spectrum of an image with a single filament is dominated by the power spectrum of the convolution kernel. As an illustration, Fig. $2 b$ displays the power spectra of images including a single model filament with either a Gaussian or a Plummer-like density profile. The red curve in Fig. 2a shows the radial profile of the Gaussian filament displayed in Fig. 1. The over-plotted black curves show the profiles of filaments featuring Plummer-like power-law wings at large radii, with power-law slopes ranging from $p=1.5$ to $p=4$. The flat inner region of each Plummer-like model filament had a constant $R_{\text {flat }}$ of $\sim 0.03 \mathrm{pc}$. An example of filament with a transverse Plummer profile is shown in Fig. A.1. Figure $2 b$ displays the power spectra corresponding to the filament profiles shown in Fig. 2a. At high angular frequencies, the power decreases exponentially following the same trend as the power spectrum of the convolution kernel. An example of a filament with a Plummerlike radial profile is shown in Fig. A.1.

\section{Diagnosing the presence of a characteristic filament width from image power spectra}

In general, dust continuum images of the diffuse, cold interstellar medium (ISM) are well described by power-law power spectra, often attributed to the turbulent nature of the flow. Herschel images are also revealing a wealth of filaments. In the following we assume that these two contributions to the emission can be treated separately, in real space

$I_{\mathrm{ISM}}(x, y)=I_{\mathrm{bkg}}(x, y)+I_{\mathrm{fil}}(x, y)$.

Under the assumption that the filaments are randomly oriented and are not correlated with the diffuse background, we can express the total power spectrum as:

$P_{\mathrm{ISM}}(k)=P_{\mathrm{bkg}}(k)+P_{\mathrm{fil}}(k)$,

where $P_{\mathrm{ISM}}(k)$ is the total power spectrum of the ISM, and $P_{\mathrm{bkg}}(k)$ and $P_{\mathrm{fil}}(k)$ represent the power spectra of the diffuse background and filament population, respectively. It is fair to assume that the power spectrum of dust images of the diffuse ISM follows a power-law, $P_{\mathrm{bkg}}(k) \propto k^{\gamma}$ with $\gamma \sim-2.7$. From Fig. 2 and Eqs. (5) and (6), it is clear that the contribution of a population of filaments with constant width $\theta_{\text {fil }}$ to the total power spectrum is not confined to a narrow range of spatial frequencies,
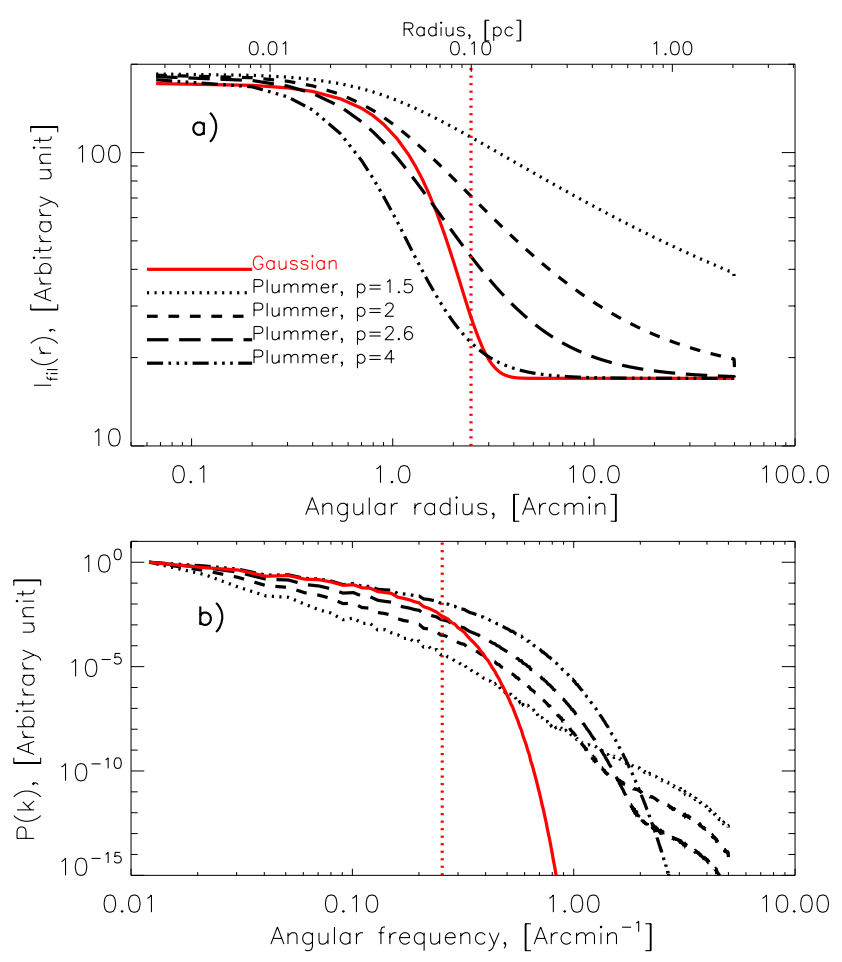

Fig. 2. Transverse profiles of several simple model filaments $(a)$ and corresponding power spectra $(b)$. Panel $a$ : red curve shows the Gaussian column density profile of the filament displayed in Fig. 1, which has a FWHM width of $0.1 \mathrm{pc}$ at a distance of $140 \mathrm{pc}$. The red vertical line marks the FWHM of the Gaussian profile. The black dashed and dotted curves display Plummer-like filament profiles with $R_{\text {flat }} \sim 0.03 \mathrm{pc}$ and logarithmic slopes $p=1.5,2,2.6$, and 4 . Panel $b$ : all power spectra were normalized to 1 at the lowest angular frequency $k_{\min }=\frac{1}{\text { Map Size }}$. The power spectra all decrease sharply at high angular frequencies, with the highest rate of decrease obtained for the Gaussian model (red curve). For the Plummer models, the rate of decrease is higher for higher $p$ values. Note the kink near $k=0.8 \mathrm{arcmin}^{-1}$ for the Plummer model with $p=1.5$, which disappears for higher $p$ values. The red vertical dashed line denotes scale $\Gamma_{\text {fil }}=0.24 \operatorname{arcmin}^{-1}$.

but rather follows a shallow power law at angular frequencies lower than $\Gamma_{\text {fil }}$.

In order to better visualize the filament contribution, we fit a power-law to the total power spectrum, $P_{\mathrm{ISM}}(k)$, and then inspect the residuals,

$\operatorname{Res}(k)=\left[P_{\text {best fit }}(k)-P_{\text {ISM }}(k)\right] / P_{\text {ISM }}(k)$,

as a function of angular frequency. To quantify the magnitude of the deviation from the best power-law fit, we use the $\chi_{\text {variance }}^{2}$ as our metric. We calculate the variance of the residuals in the vicinity of $k_{\text {fil }}$ where the contribution of filament power is expected to be maximum ${ }^{1}$. We define the variance as

$\chi_{\text {variance }}^{2}=\Sigma_{k_{\min }}^{1.5 k_{\text {fil }}} \operatorname{Res}(k)^{2} / N_{\text {freq }}$,

where $\operatorname{Res}(k)$ is the residual at angular frequency $k$ defined by Eq. (9), and $N_{\text {freq }}$ is the total number of frequency modes ${ }^{2}$ between $k_{\min }$ and $1.5 \times k_{\mathrm{fil}}$. The upper bound in the above summation is set to $1.5 \times k_{\text {fil }}$ in a conservative sense, since the

\footnotetext{
1 Note that $P_{\text {fil }}(k)$ in Fig. 2 is almost flat at $k<k_{\text {fil }}$ and drops rapidly at $k>k_{\text {fil }}$.

$2 k_{\min }=\frac{1}{\text { Map Size }}$ is the minimum angular frequency considered in the
} power spectrum. 
power spectrum of constant-width filaments drops at $k>k_{\text {fil }}$ (see Fig. 2b), and $\operatorname{Res}(k)$ is therefore dominated by the diffuse ISM contribution for $k>k_{\mathrm{fil}}$. In principle, the image power spectrum of a scale-free ISM will have residuals close to zero, and any significant deviation of the residuals from zero at $k<k_{\text {fil }}$ will be primarily due to the power spectrum of filaments (see Eq. (8)). Thus, one expects $\chi_{\text {variance }}^{2} \propto \sum\left[P_{\text {best fit }}(k)-P_{\text {ISM }}(k)\right]^{2} \propto$ $\sum P_{\text {fil }}(k)^{2}$. Simple dimensional analysis of the Parseval relation between $P_{\text {fil }}(k)^{2}$ and $\left|I_{\text {fil }}(x, y)\right|^{2}$ provides deeper insight into the connection between $\chi_{\text {variance }}^{2}$ and observable parameters of the filament population:

$\left[\chi_{\text {variance }}^{2}\right]=\left[\sum P_{\text {fil }}(k)^{2}\right]=\left[\int\left|I_{\text {fil }}(x, y)\right|^{2} \mathrm{~d} x \mathrm{~d} y\right]^{2}$,

where $[x]$ in square brackets denotes the dimension of quantity $x$. Dimensional analysis thus suggests that $\chi_{\text {variance }}^{2}$ must be a function of $\delta_{\mathrm{c}}^{2} A_{\mathrm{fil}}$ :

$\chi_{\text {variance }}^{2}=\phi\left(\delta_{\mathrm{c}}^{2} A_{\mathrm{fil}}\right)$.

The $\delta_{\mathrm{c}}^{2}$ dependence comes from exploiting Eqs. (1) and (3), while the area filling factor $A_{\text {fil }} \equiv S_{\text {fil }} / S$ dependence comes from the fact that only the effective area $S_{\text {fil }}$ over which filaments are distributed contribute to the integral on the right-hand side of Eq. (11). For low $A_{\text {fil }}$ and $\delta_{\mathrm{c}}$ the variance is very small, while for high $A_{\text {fil }}$ and/or high $\delta_{\mathrm{c}}$ the variance metric can be very high. We will explore the $\chi_{\text {variance }}^{2}-\delta_{\mathrm{c}}^{2} A_{\text {fil }}$ parameter space in more detail in Sect. 7 below.

The magnitude/amplitude of excess power in the ISM power spectrum $P_{\mathrm{ISM}}(k)$ relative to the best-fit power law model power spectrum at a characteristic frequency $k_{\mathrm{fil}}$ depends upon the combined effect of the mean filament contrast in the image and the fractional area covered by the filaments, $A_{\text {fil }}$. The area filling factor $A_{\text {fil }}$ can be expressed as $A_{\text {fil }}=\Sigma_{i=1}^{N_{\text {fil }}} L_{\mathrm{i}} \times W_{\text {fil }} / S$, where $L_{i}$ is the length of the $i$ th filament, $W_{\text {fil }}$ the transverse filament width $(\sim 0.1 \mathrm{pc}), S$ the total area coverage of the image being analyzed, and $N_{\text {fil }}$ the total number of filaments in the image.

\section{Power spectrum of the Polaris Herschel data}

In this section, we analyze the Herschel/SPIRE image of the Polaris Flare cloud at $250 \mu \mathrm{m}$ (Miville-Deschênes et al. 2010; Ward-Thompson et al. 2010; see also Schneider et al. 2013), which covers an area of $3.0^{\circ} \times 3.3^{\circ}$ and is shown in Fig. 3a) at the native (diffraction-limited) beam resolution of $18^{\prime \prime} .2$. For our analysis, a pixel size of $6^{\prime \prime}$ was adopted ${ }^{3}$.

The Polaris Flare image displays a spectacular distribution of low column density filaments. All of these filaments are thermally subcritical. The mean peak surface brightness contrast of these filaments over the local background is around $\left\langle\delta_{\mathrm{c}}\right\rangle \sim 0.9$, but the filaments occupy only a small fraction $\sim 2 \%$ of the total surface area, leading to $\delta_{\mathrm{c}}^{2} A_{\mathrm{fil}} \sim 0.016$.

To first order, the transverse structure of the Polaris filaments is well described by Gaussian profiles with a FWHM of $\sim 0.1 \mathrm{pc}$ (assuming a distance $\sim 140 \mathrm{pc}$ for the Polaris cloud) (Arzoumanian et al. 2011, 2019). Miville-Deschênes et al. (2010) carried out a power spectrum analysis for the Polaris image over spatial scales ranging from $0.01 \mathrm{pc}$ to $10 \mathrm{pc}$. The power spectrum revealed a continuous power-law, $P(k) \propto k^{\gamma}$, with an exponent of $\gamma=-2.65$ down to the scale of the

\footnotetext{
3 A zero-level offset of $16.8 \mathrm{MJy} / \mathrm{sr}$ was also added to the image based on a comparison with Planck and IRAS data.
}
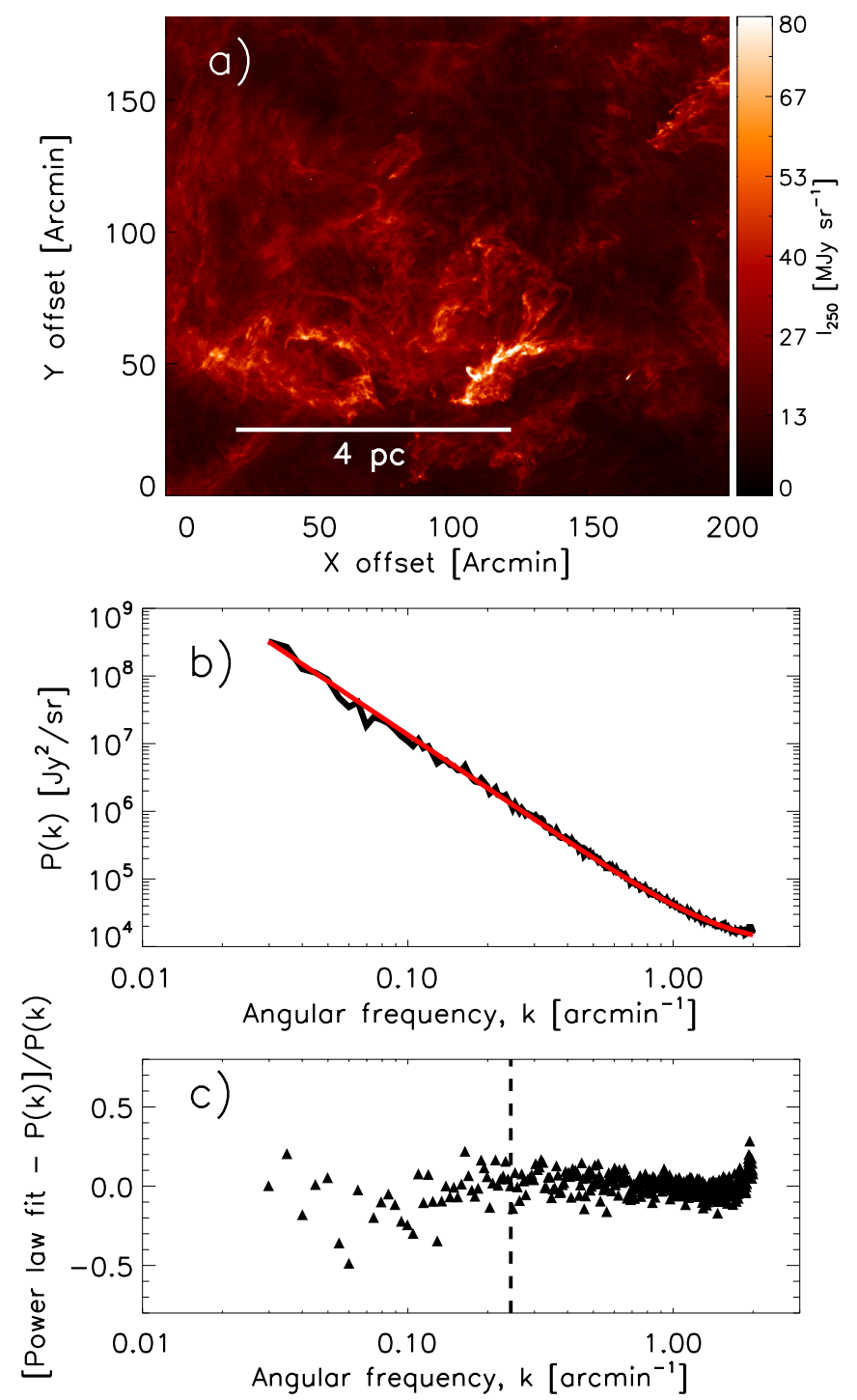

Fig. 3. Panel a: Herschel/SPIRE $250 \mu \mathrm{m}$ emission image of a part of the Polaris cloud. The HPBW angular resolution is 18,2 . Panel $b$ : noisesubtracted and beam-corrected power spectrum of the image shown in panel a over the range of angular frequencies $0.025 \mathrm{arcmin}^{-1}<$ $k<2 \operatorname{arcmin}^{-1}$. The red curve shows the best fit power-law model over this frequency range, which takes the form $P_{\text {sky }}(k)=A_{\mathrm{ISM}} k^{\gamma}+P_{0}$ with $\gamma=-2.63 \pm 0.1$. Panel $c$ : residuals between the best-fit powerlaw model and the power spectrum data points (triangle symbols). The $\chi_{\text {Variance }}^{2}$ (see Eq. (10)) of the residuals is $\sim 0.03$. The vertical dashed line marks the angular frequency $k_{\text {fil }} \sim\left(0.6 / \theta_{\text {fil }}\right)$ corresponding to a characteristic filament width of $\sim 0.1 \mathrm{pc}$.

beam, suggesting a scale-free image. Following the same scheme as Miville-Deschênes et al. (2010), we derived the power spectrum of a sub-field ${ }^{4}$ of Polaris shown in Fig. 3. Prior to computing of power spectrum, we apodized the edges of the image by a sine function to ensure smooth periodic boundary condition. After subtracting the noise power spectrum level estimated from the mean power at angular frequencies $k>3.5 \mathrm{arcmin}^{-1}$, we corrected for convolution effects by

\footnotetext{
4 In order to capture the maximum rectangular area within the Polaris image for easier computation of the power spectrum, we rotated the original map in equatorial coordinate by $13.6^{\circ}$ clockwise about its center and extracted the largest area excluding turn-around data points near the edges of the field.
} 
dividing the observed power spectrum by the power spectrum of the Herschel telescope beam at $250 \mu \mathrm{m}$ (Martin et al. 2010) obtained from scans of Neptune. In order to derive the power spectrum slope, we then fitted a power-law model of the form $P_{\text {sky }}(k)=A_{\text {ISM }} k^{\gamma}+P_{0}$ to the corrected power spectrum over the range of angular frequencies $0.025 \mathrm{arcmin}^{-1}<k<2 \operatorname{arcmin}^{-1}$, as described in Miville-Deschênes et al. (2010). In Fig. 3b, we show the power spectrum of the Polaris image over the range of angular frequencies used in the power-law fit. The red curve represents the best-fit power law with $\gamma=-2.63 \pm 0.1$, which is very close to the $\gamma=-2.65 \pm 0.1$ value obtained by Miville-Deschênes et al. (2010). Visual inspection shows that there is no clear spectral signature of a characteristic scale embedded in the observed power spectrum. Figure $3 \mathrm{c}$ shows the residuals $\left[P_{\text {Best-fit }}(k)-P_{\text {Polaris }}(k)\right] / P_{\text {Polaris }}(k)$ as a function of angular frequency. Any significant kink or distortion in the power spectrum due to the presence of a characteristic scale should in principle be captured as a significant deviation from zero in the plot of residuals. The plot of residuals for the Polaris Flare image (Fig. 3c) does not exhibit such a deviation.

In order to critically analyze this finding, we performed a suite of numerical experiments by injecting synthetic filaments separately into 1) the original Herschel/SPIRE $250 \mu \mathrm{m}$ image of Polaris, and 2) the filament-subtracted image obtained after applying the getfilaments algorithm (Men'shchikov 2013) to the Herschel/SPIRE image to remove most of the real filamentary structures. We repeated the same power spectrum analysis as described above on both sets of modified Herschel images (see Sects. 4.2 and 4.3 below). For this analysis, we preferred to use SPIRE $250 \mu \mathrm{m}$ data rather than $18^{\prime \prime} .2$ column density images produced from the combination of Herschel data at $160 \mu \mathrm{m}$ to $500 \mu \mathrm{m}$ (cf. Palmeirim et al. 2013) because the former are less affected by noise and better behaved from a power-spectrum point of view (cf. Miville-Deschênes et al. 2010).

\subsection{Construction of an image with synthetic filaments}

To create a synthetic filament image, the first step was to generate a map of randomly oriented 1D delta line functions as described in Sect. 2. Then, we convolved this initial synthetic map with a Gaussian kernel such that the projected spatial FWHM width of the kernel was $0.1 \mathrm{pc}$ as described in Sect. 2. When creating synthetic filaments we neglected the fluctuations observed along real Herschel filaments (Roy et al. 2015), because the contrast of these fluctuations above the average filament is $\ll 1$, and also the area filling factor of these fluctuations is very small. To maximize the effect of a characteristic width in our simulations, we fixed the FWHM width of the Gaussian filaments to a strictly constant value. We controlled the contrast parameter $C$ of each filament by measuring the local background emission in the close vicinity of the filament within the background image. The distribution of the contrast parameter was chosen to reflect the observed distribution in each region. In the simulation, we varied the angular length of the filaments randomly between a minimum of $30 \times 18^{\prime \prime} \cdot 2=546^{\prime \prime}$ and a maximum of $70 \times 18^{\prime \prime} .2=1274^{\prime \prime}$, corresponding to 0.4 to $0.9 \mathrm{pc}$ at $d=140 \mathrm{pc}$. Figure $4 \mathrm{a}$ shows one such realization including a population of synthetic filaments with a lognormal distribution of contrasts in the range $0.3<\delta_{\mathrm{c}}<$ 2.0, co-added to the original map of Polaris. In this example, the population of synthetic filaments has an area filling factor $A_{\text {fil }} \sim 3.2 \%$.

\subsection{Effect of synthetic filaments on the power spectrum of the Polaris image}

Next, we investigated the effect of synthetic filaments on the power spectrum of the Polaris original image on one hand, and the power spectrum of the filament-subtracted Polaris image on the other hand. First, we discuss the case of the Polaris original image.

Figure $4 \mathrm{~b}$ shows the total power spectrum of the Polaris image in Fig. 4a, which includes a population of synthetic filaments with a log-normal distribution of contrasts, $\delta_{\mathrm{c}}$. In Fig. 4, the range of contrast values in the synthetic distribution varied in the range $0.3<\delta_{\text {c }}<2.0$ with a peak at $\delta_{\text {peak }} \sim 0.9$. The weighted average of the contrast over the length of the simulated filaments is $\left\langle\delta_{\mathrm{c}}\right\rangle \sim 0.85$. In Fig. 4a, the synthetic filaments are clearly visible against their local background. The best power-law fit to the power spectrum (red curve) has a logarithmic slope $\gamma=-2.7 \pm 0.1$, slightly steeper than the slope of the Polaris original image. The vertical dashed line marks the angular frequency, $k_{\mathrm{fil}}=\Gamma \sim\left(0.6 / \theta_{\mathrm{fil}}\right) \sim 0.24 \mathrm{arcmin}^{-1}$, corresponding to the characteristic angular width of the synthetic filaments, $\theta_{\text {fil }}=147^{\prime \prime}$ (i.e., $0.1 \mathrm{pc}$ at $d=140 \mathrm{pc}$ ). Comparison of Figs. $4 \mathrm{~b}$ and $3 \mathrm{~b}$ shows that the synthetic filaments contribute an insignificant amount of power around $k_{\text {fil }}=0.24 \operatorname{arcmin}^{-1}$, which can hardly be detected without prior knowledge of the power spectrum of the original ISM image. Figure $4 \mathrm{c}$ plots the normalized residuals between the best power-law fit and the power spectrum data, $\left[P_{\text {Best-fit }}(k)-P_{\text {Polaris }}(k)\right] / P_{\text {Polaris }}(k)$, as a function of angular frequency. These residuals (red filled circles) can be compared with the residuals obtained with the original image, represented by black triangles in both Figs. $3 \mathrm{c}$ and $4 \mathrm{c}$. Again, no clear signature of the presence of synthetic filaments can be detected despite the fact that they have a characteristic width.

Now let us investigate the power spectrum of each component more closely to understand the absence of any detectable signature in the total power spectrum. The blue curve and the green dashed curve in Fig. 4b show the power spectrum of the synthetic filament image and that of the original image, respectively. Note that the power spectrum of the filament image, $P_{\text {fil }}(k)$, is lower than the power spectrum $P_{\text {Polaris }}(k)$ at $k=k_{\mathrm{fil}}$ by a factor of $\sim 5$. This is because the population of synthetic filaments only have moderate area filling factor $\left(A_{\text {fil }} \sim 3.2 \%\right)$ and contrast $\left(\left\langle\delta_{\mathrm{c}}\right\rangle \sim 0.85\right)$. In this experiment, the product of the area filling factor with the square of the column density contrast $\left(A_{\mathrm{fil}} \delta_{\mathrm{c}}^{2} \sim 0.02\right)$ was in agreement with the real filaments observed in Polaris (which have $A_{\text {fil }}\left\langle\delta_{\mathrm{c}}\right\rangle^{2} \sim 0.01-\mathrm{cf}$. Arzoumanian et al. 2019). The additional power introduced by the synthetic filaments is not localized in the vicinity of $k_{\mathrm{fil}}$ but rather spread out at angular frequencies $k \lesssim k_{\text {fil }}$, following a shallow power-law, whereas the power at high angular frequencies at $k>k_{\text {fil }}$ drops sharply. Given the choice of $\delta_{\mathrm{c}}$ and $A_{\text {fil }}$ made here, the relative contribution of filaments to the total power spectrum, $P_{\text {fil }}(k) / P_{\text {Polaris }}(k)$ is highest in the vicinity of $k_{\text {fil }}$, but too small to create any detectable feature in the power spectrum.

When the contrast and/or filling factor of the synthetic filaments is gradually increased, the spectral imprint in the resulting power spectrum becomes more and more pronounced. Figure B.2a shows a simulated image including a population of synthetic $0.1 \mathrm{pc}$ filaments with contrast $\delta_{\mathrm{c}} \sim 1.1$ and area filling factor $A_{\text {fil }} \sim 7.2 \%$. This is quite an extreme scenario for a non-star-forming molecular cloud with low column density such as Polaris. Figure B.2b shows the corresponding power spectra, which should be compared to those in Fig. 4b. It can be seen that the amplitude of the synthetic power spectrum in 

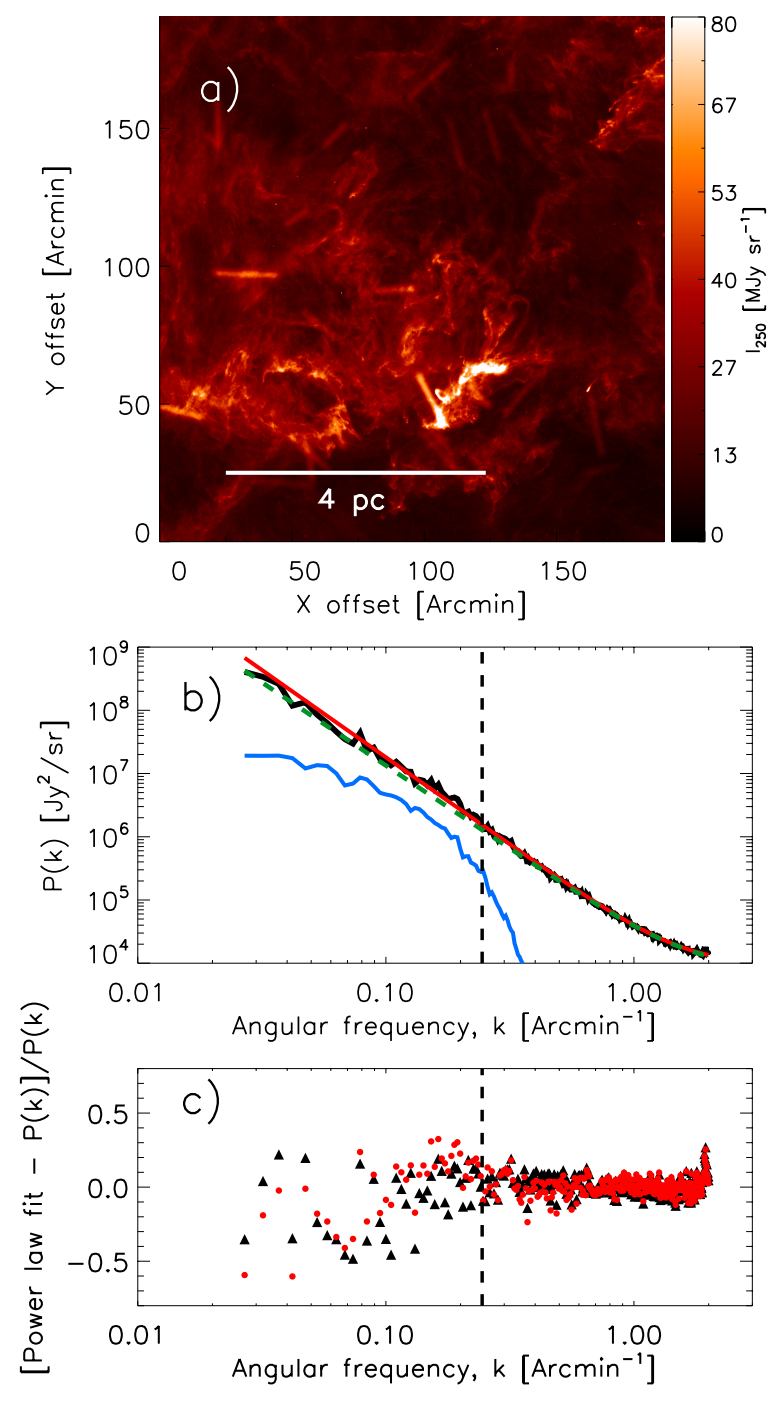

Fig. 4. Same as Fig. 3 but for a $250 \mu \mathrm{m}$ image with an additional population of synthetic filaments. The population of synthetic filaments has a lognormal distribution of contrasts in the range $0.3<\delta_{\mathrm{c}}<2.0$ with a broad peak around $\delta_{\text {peak }} \sim 0.9$. The overall area filling factor of the synthetic filaments is $A_{\text {fil }} \sim 3 \%$ and the $\delta_{\mathrm{c}}^{2} A_{\text {fil }}$ parameter (see Sect. 3) is 0.023 . Panel $b$ : solid black curve shows the total power spectrum of the original Polaris image plus synthetic filaments. The best-fit powerlaw (red curve) has $\gamma=-2.7 \pm 0.1$, slightly steeper than the slope of the original power spectrum of the Polaris image shown by the dashed green curve. The blue curve is the power spectrum of the image containing only synthetic filaments. Panel $c$ : black triangles are the same as in Fig. $3 \mathrm{c}$ and the red dots show the residuals between the best-fit powerlaw model and the power spectrum of the image including synthetic filaments. The $\chi_{\text {variance }}^{2}$ of the residuals between $k_{\min }<k<1.5 k_{\mathrm{fil}}$ is 0.037 . The vertical dashed line marks the angular frequency $k_{\text {fil }} \sim\left(0.6 / \theta_{\text {fil }}\right)$ corresponding to the characteristic $\sim 0.1 \mathrm{pc}$ width of the synthetic filaments.

Appendix B (with contrast $\left\langle\delta_{\mathrm{c}}\right\rangle \sim 1.1$ and $A_{\mathrm{fil}} \sim 7.2 \%$ ) is higher by a factor of 3 to 4 than the amplitude of the power spectrum of Fig. $4 \mathrm{~b}$ (with contrast $\left\langle\delta_{\mathrm{c}}\right\rangle \sim 0.85$ and $A_{\text {fil }} \sim 3.2 \%$ ), mostly due to the increase in the combination of contrast parameter and area-filling factor $\left\langle\delta_{\mathrm{c}}\right\rangle^{2} A_{\text {fil }}$ between the two simulations $\left[\sim(1.1 / 0.85)^{2} \times(7.2 / 3.2)(\sim 3.7)\right]$. The red curve in Fig. B.2b shows the best power-law fit which has a logarithmic slope $\gamma=-2.96 \pm 0.1$. At $k \lesssim k_{\text {fil }}$, there is a significant enhancement of power due to the fact the synthetic filament power spectrum $P_{\text {fil }}(k)$ is now comparable to the Polaris power spectrum
$P_{\text {Polaris }}(k)$ at $k \lesssim k_{\text {fil }}$. Accordingly, in this case, the residuals between the best power-law fit and the total power spectrum data depart significantly from zero at $k \lesssim k_{\text {fil }}$ (cf. Fig. B.2c). It is to be borne in mind, however, that the population of synthetic filaments used in Appendix B have much higher contrast and area filling factor than the actual filaments of the Polaris cloud (compare Figs. B.2a and 3a).

\subsection{Effect of synthetic filaments on the power spectrum of the filament-subtracted image}

So far we have explored the response of the power spectrum to a synthetic population of filaments injected into the original Herschel image, which itself includes emission from real filamentary structures. It is instructive to assess the extent to which the real filaments present in the image may reduce the relative contribution of synthetic filaments. In order to evaluate this we adopted two approaches - first, we subtracted the emission of at least the most prominent real filamentary structures from the Polaris $250 \mu \mathrm{m}$ image using the getfilaments algorithm (Men'shchikov 2013, see Fig. B.1b for the resulting filamentsubtracted image) and then repeated the same experiment as described in Sect. 4.1. Second, we examined the effect of filaments embedded in a typical scale-free synthetic cirrus images (see Appendix C). In order to be consistent, we used the same population of synthetic filaments as in Sects. 4.1 and 4.2.

Figure 5 summarizes the effect of the synthetic 0.1 pc filaments on a background image which is essentially devoid of real filamentary structures. Although the logarithmic power-spectrum slope of the background image is shallower $\left(\gamma_{\mathrm{bkg}}=-2.5\right)$ than that of the Polaris original image $\left(\gamma_{\mathrm{obs}}=-2.7\right)$, the overall morphology remains the same. In particular, the power spectrum of the synthetic filament component is still significantly lower than the total power spectrum of the background image, even though the power arising from real filaments has been subtracted from that image. The $\chi_{\text {variance }}^{2}$ of the filament-subtracted background image is 0.04 , very close the $\chi_{\text {variance }}^{2}$ value obtained for the Polaris original image. Moreover, there is still no clear signature of the presence of synthetic filaments in the residuals plot (Fig. 5c). Similar conclusions were reached in Appendix $\mathrm{C}$ in the case of synthetic filaments added to a purely synthetic background image.

\section{Exploring the parameter space with simulations in the Aquila cloud}

The Aquila molecular cloud harbors a statistically significant number of filaments with a wide range of filament column density contrasts (Könyves et al. 2015; Arzoumanian et al. 2019), allowing us to derive a realistic distribution of contrasts which can then be used for constructing more realistic populations of synthetic filaments.

\subsection{Observed filament properties in Aquila}

In contrast to the Polaris cloud, the Aquila molecular cloud is an active star forming complex at a distance ${ }^{5}$ of $260 \mathrm{pc}$, including several supercritical filaments (André et al. 2010; Könyves et al. 2015). Figure 6a shows the Herschel/SPIRE $250 \mu \mathrm{m}$ image of the

5 The distance of the Aquila cloud is uncertain, with values ranging from $260 \mathrm{pc}$ to $414 \mathrm{pc}$ in the literature. Assuming the upper distance value would push $k_{\text {fil }}$ toward higher angular frequencies in Fig. $6 \mathrm{~b}$ and $\mathrm{c}$, making the detection of the $0.1 \mathrm{pc}$ scale even more difficult in the power spectrum. 

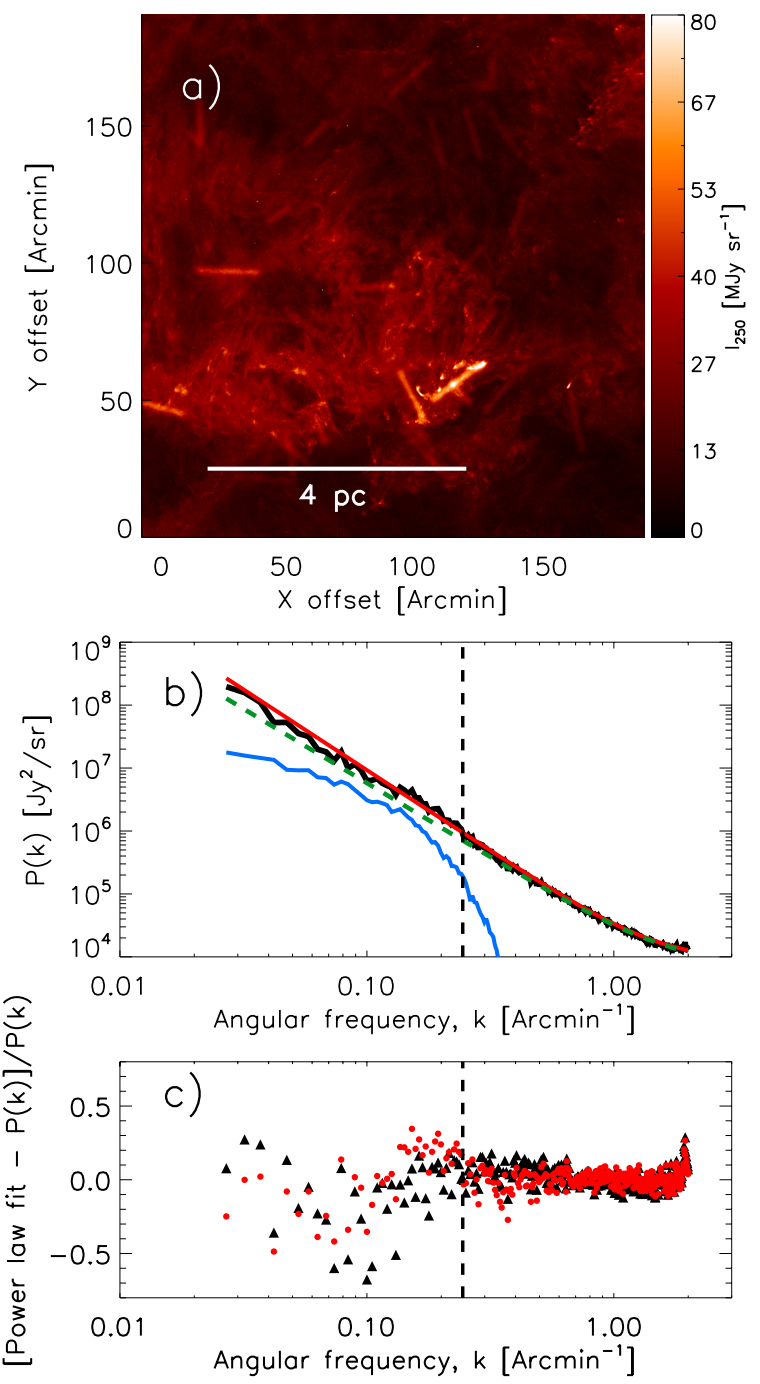

Fig. 5. Same as Fig. 4 for synthetic filaments added to a filamentsubtracted image of Polaris obtained with getfilaments (Men'shchikov 2013). The population of synthetic filaments is the same as that in Fig. 4. Panel $b$ : green dashed line shows the best power-law fit to the power spectrum of the filament-subtracted background image of Polaris (with no synthetic filaments), which has a slope of $\gamma=-2.4 \pm 0.1$. The solid black curve shows the total power spectrum of the filament-subtracted image plus synthetic filaments. The best-fit power-law (red curve) has a slope of $\gamma=-2.5 \pm 0.1$, slightly steeper than the slope of the power spectrum of the filament-subtracted background image (dashed green curve). The blue curve is the power spectrum of the image containing only synthetic filaments. Panel $c$ : black triangles are the same as in Fig. $3 \mathrm{c}$ and the red dots show the residuals between the best-fit power-law model and the power spectrum of the image including synthetic filaments. The $\chi_{\text {variance }}^{2}$ of the residuals between $k_{\min }<k<1.5 k_{\text {fil }}$ is 0.034 . The vertical dashed line marks the angular frequency $k_{\mathrm{fil}} \sim\left(0.6 / \theta_{\mathrm{fil}}\right)$ corresponding to the characteristic $\sim 0.1 \mathrm{pc}$ width of the synthetic filaments.

Aquila cloud, which covers a projected sky area of $3.4^{\circ} \times 3.2^{\circ}$. The corresponding power spectrum is shown in Fig. $6 b$.

As part of a systematic analysis of filament properties in nearby clouds based on HGBS data, Arzoumanian et al. (2019) took a census of filamentary structures in Aquila. They obtained a distribution of filament column density contrasts which can be conveniently approximated by the two-segment power law shown in Fig. 7: $\mathrm{d} N / \mathrm{d} \log \left(\delta_{\mathrm{c}}\right) \sim$ const for $0.3 \leq \delta_{\mathrm{c}} \leq 1$, and $\mathrm{d} N / \mathrm{d} \log \left(\delta_{\mathrm{c}}\right) \sim \delta_{\mathrm{c}}^{-1.5}$ for $1 \leq \delta_{\mathrm{c}} \leq 4$. This observed distribution of filaments contrasts has a peak around $\delta_{\mathrm{c}}^{\text {peak }} \sim 1$ and spans
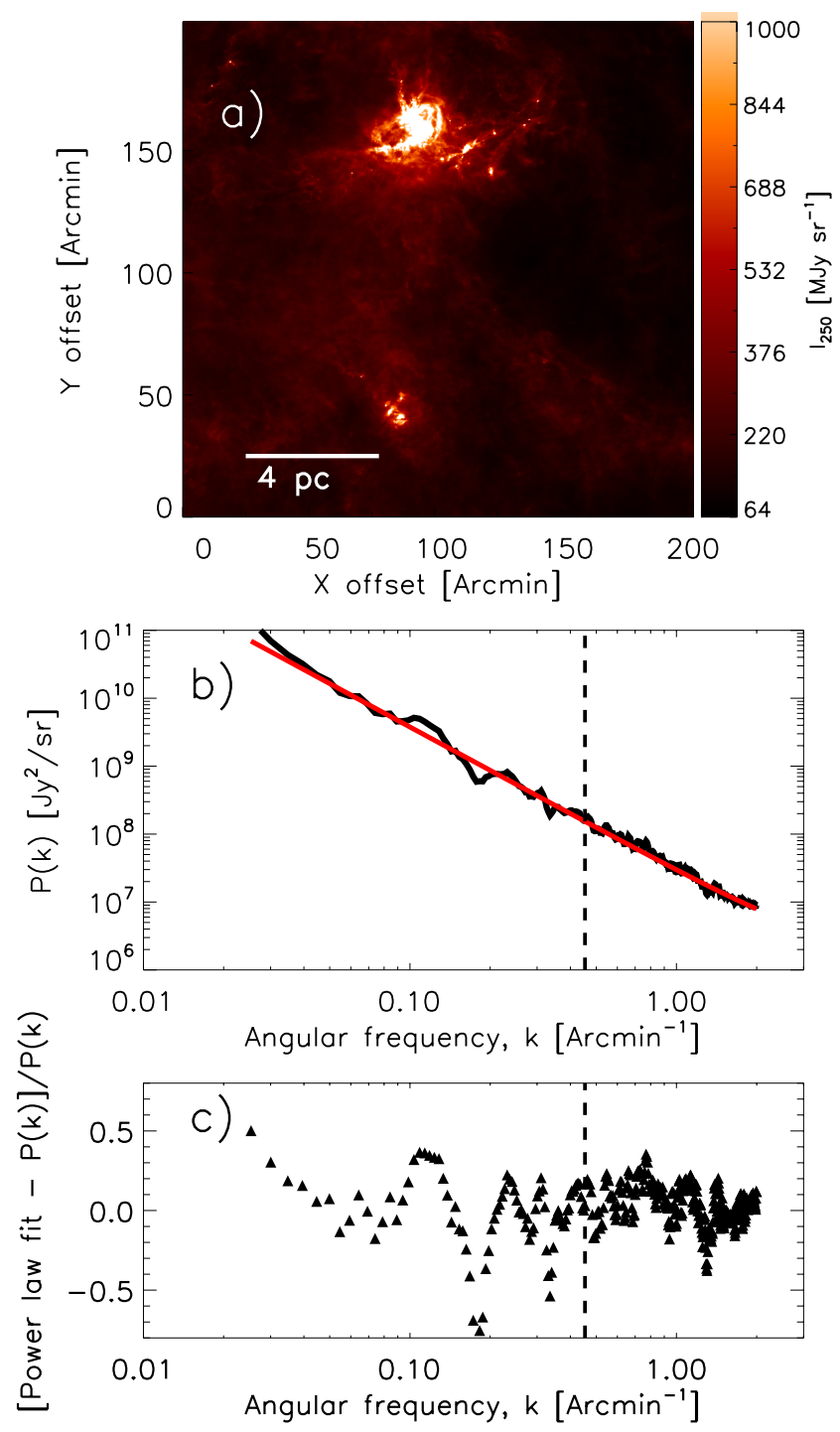

Fig. 6. Panel a: Herschel/SPIRE $250 \mu \mathrm{m}$ image of the Aquila cloud at the native resolution of $18^{\prime \prime} .2$. Panel $b$ : noise-subtracted and beamcorrected power spectrum of the image shown in panel a over the range of angular frequencies $0.025 \mathrm{arcmin}^{-1}<k<2 \operatorname{arcmin}^{-1}$ (black curve). The red curve shows the best fit power-law model over this frequency range, which has a logarithmic slope $\gamma=-2.26 \pm 0.1$. The vertical dashed line marks the angular frequency $k_{\text {fil }} \sim\left(0.6 / \theta_{\text {fil-width }}\right) \sim$ $0.45 \mathrm{arcmin}^{-1}$ corresponding to a filament width of $\theta_{\text {fil-width }}=79^{\prime \prime}$ (FWHM), i.e., $0.1 \mathrm{pc}$ at a distance of $260 \mathrm{pc}$. Panel $c$ : residuals between the best power-law fit and the power spectrum data points (triangle symbols). The $\chi_{\text {Variance }}^{2}$ of the residuals between $k_{\min }<k<1.5 k_{\text {fil }}$ is $\sim 0.045$.

a broad range from low $\delta_{\mathrm{c}} \sim 0.3$ values to fairly high $\delta_{\mathrm{c}} \sim 4$ values. The weighted average column density contrast of the filaments observed in Aquila is $\left\langle\delta_{\mathrm{c}}\right\rangle \sim 1$, and their area filling factor is $A_{\text {fil }} \sim 3 \%$. While the census of filaments obtained by Arzoumanian et al. (2019) may be affected by incompleteness issues for low-contrast ${ }^{6}\left(\delta_{\mathrm{c}} \ll 1\right)$ filaments, it should be essentially complete for high-contrast $\left(\delta_{\mathrm{c}} \gtrsim 1\right)$ supercritical filaments.

6 Given the fact that the amplitude of a power spectrum $\propto \delta_{\mathrm{c}}^{2}$, undetected filaments (with low contrasts) below the completeness level will not have any significant effect on the net amplitude of synthetic filaments power spectrum. 


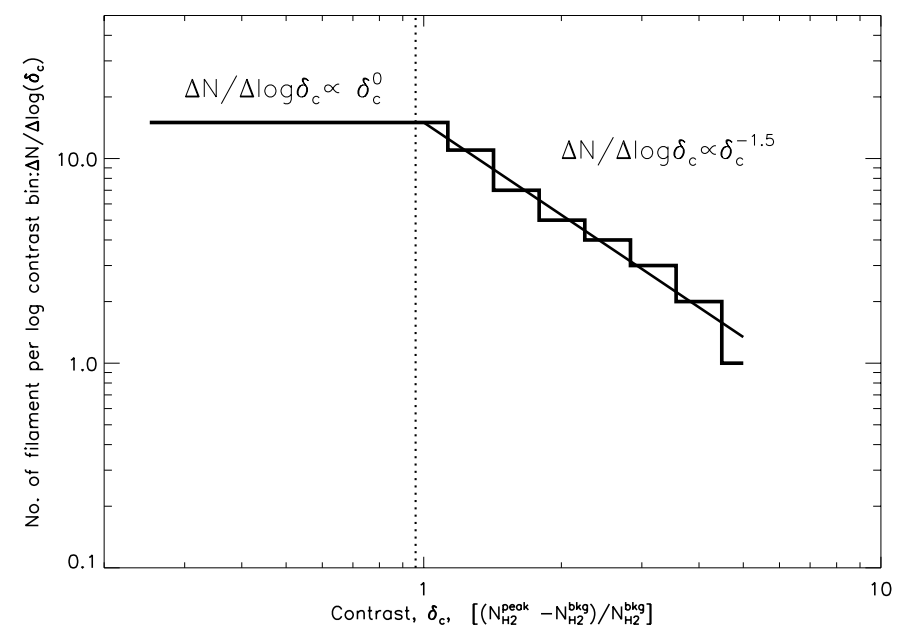

Fig. 7. Two-segment power-law approximation (black solid lines) to the distribution of filament column density contrasts observed in the Aquila molecular cloud (Arzoumanian et al. 2019): $\mathrm{d} N / \mathrm{d} \log \left(\delta_{\mathrm{c}}\right) \sim$ const for $0.3 \leq \delta_{\mathrm{c}} \leq 1$, and $\mathrm{d} N / \mathrm{d} \log \left(\delta_{\mathrm{c}}\right) \sim \delta_{\mathrm{c}}^{-1.5}$ for $1 \leq \delta_{\mathrm{c}} \leq 4$. The vertical dotted line marks the average filament contrast $\left\langle\delta_{\mathrm{c}}\right\rangle \sim 1$. The overplotted histogram shows the distribution of column density contrasts for the population of 100 synthetic filaments used in the simulation of Sect. 4.

\subsection{Effect of a synthetic population of filaments on the power spectrum}

Using a methodology similar to that employed in Sect. 4 for Polaris, we added a population of synthetic filaments with fixed $0.1 \mathrm{pc}$ width to a filament-subtracted Herschel image of the Aquila region at $250 \mu \mathrm{m}$. The distribution of column density contrasts $^{7}$ for the synthetic filaments was constructed to be consistent with observations and is represented by the histogram in Fig. 7. The weighted mean contrast of the whole population of synthetic filaments was $\left\langle\delta_{\mathrm{c}}\right\rangle \sim 0.96$. Like in the Polaris case, the background image was obtained from the Herschel/SPIRE $250 \mu \mathrm{m}$ of the Aquila cloud image after removing observed filaments using the getfilaments algorithm (Men'shchikov 2013). The resulting synthetic image is shown in Fig. 8a. Figure 8b shows the power spectrum of each component in the synthetic image: the blue curve corresponds to the contribution of the synthetic filament distribution, while the black curve is the total power spectrum of the Aquila background plus filament image.

It can be seen in Fig. $8 \mathrm{~b}$ that the amplitude of the power spectrum arising from the population of synthetic filaments (blue curve) is lower than the amplitude of the power spectrum of the Aquila original image (green dashed curve) by a factor of $\sim 5$ at $k \sim k_{\text {fil }} \sim\left(0.6 / \theta_{\text {fil }}\right) \sim 0.45 \operatorname{arcmin}^{-1}$, corresponding to the characteristic angular width of the synthetic filaments, $\theta_{\text {fil }}=79^{\prime \prime}$ (i.e., $0.1 \mathrm{pc}$ at $d=260 \mathrm{pc}$ ). Clearly, the power contribution of the synthetic filaments is not strong enough to be detected in the power spectrum. The residuals of the best power-law fit with respect to the power spectrum of the Aquila original image are shown as black triangles in Fig. 8c as a function of angular frequency. The red solid circles in Fig. 8c represent similar residuals for the Aquila background plus synthetic filament image. Based on this simulation, we conclude that the injection of a population

\footnotetext{
The column density contrasts of cold molecular filaments are somewhat higher than their surface brightness contrasts at $250 \mu \mathrm{m}$. To be on the conservative side, we used the observed distribution of column density contrasts for constructing synthetic filaments in the $250 \mu \mathrm{m}$ images. The actual surface brightness contrasts are actually lower than what we assumed here.
}
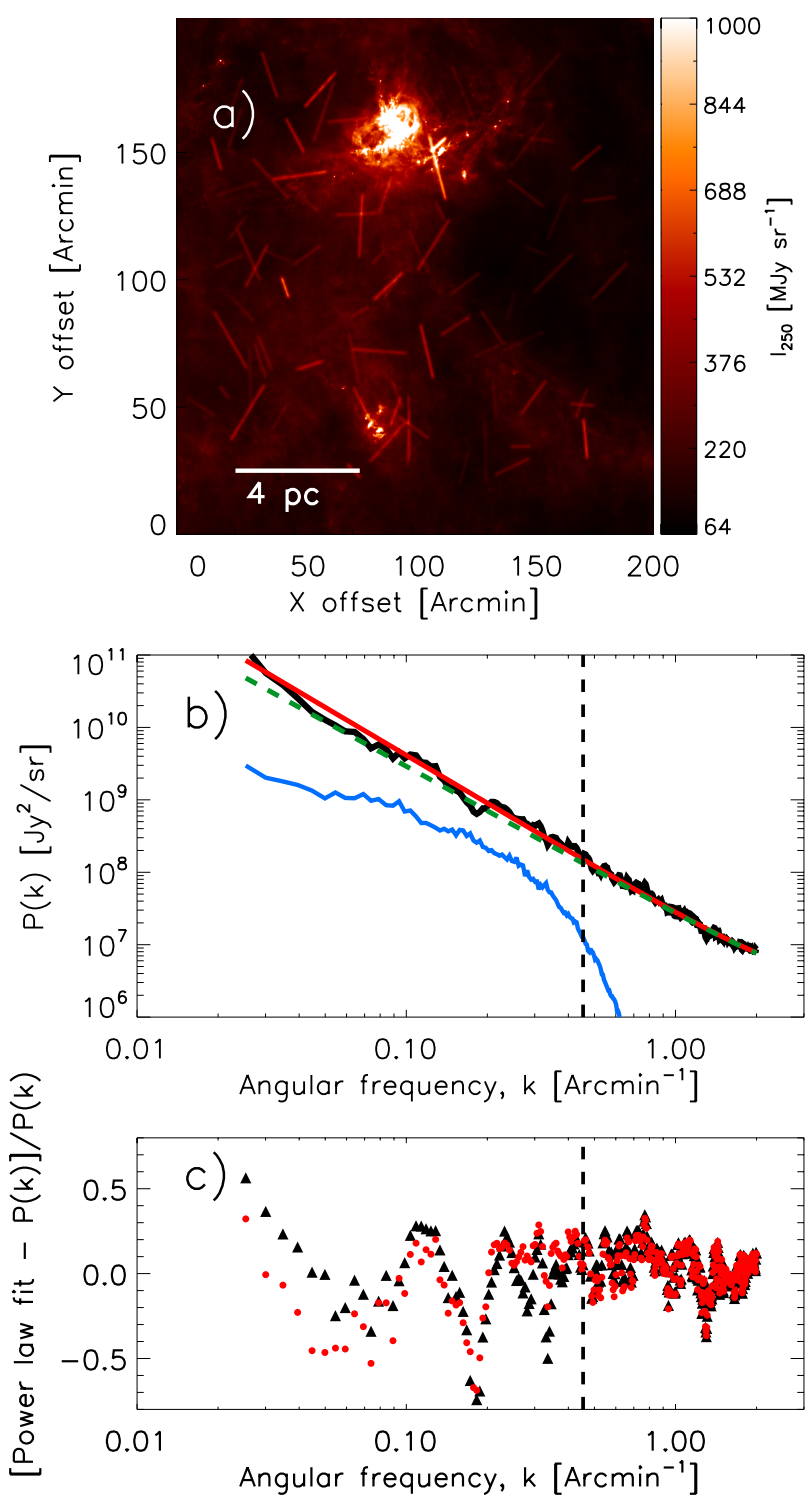

Fig. 8. Same as Fig. 6 but for a simulated image including a population of synthetic filaments with a realistic distribution of column density contrasts (see Fig. 7) added to a filament-subtracted image of the Aquila cloud. Panel $a$ : simulated image. The weighted average contrast $\left\langle\delta_{c}\right\rangle$ of the distribution of synthetic filaments is 0.96 and the total area covering factor $A_{\text {fil }}$ is $5.5 \%$, leading to $\delta_{\mathrm{c}}^{2} A_{\mathrm{fil}} \sim 0.051$. Panel $b$ : power spectrum of the simulated image (black solid curve). The red curve corresponds to the best power-law fit with $\gamma=-2.3 \pm 0.1$. For comparison, the best power-law fit to the power spectrum of the Aquila original image is over-plotted as a green dashed line. Panel $c$ : residuals between the best power-law fit and the power spectrum of the simulated image (red solid circles). The $\chi_{\text {Variance }}^{2}$ of the residuals is $\sim 0.054$ For comparison, the black filled triangles show similar residuals for the Aquila original image (cf. Fig. 6c).

of synthetic filaments with a distribution of column density contrasts similar to that observed in the real Aquila image does not have any significant effect on the shape of the power spectrum.

In Appendix B, we also explore a more extreme case where the distribution of column density contrasts for the injected synthetic filaments is similar in shape to the distribution shown in Fig. 7, but with higher mean contrast $\left\langle\delta_{\mathrm{c}}^{\text {peak }}\right\rangle=2.7$ and maximum contrast $\delta_{\mathrm{c}}^{\max }=15$. In this extreme case, the population of synthetic filaments is strong enough to produce a detectable signature in the resulting power spectrum. 
A. Roy et al.: Effect of a characteristic filament width on the power spectrum of cloud images
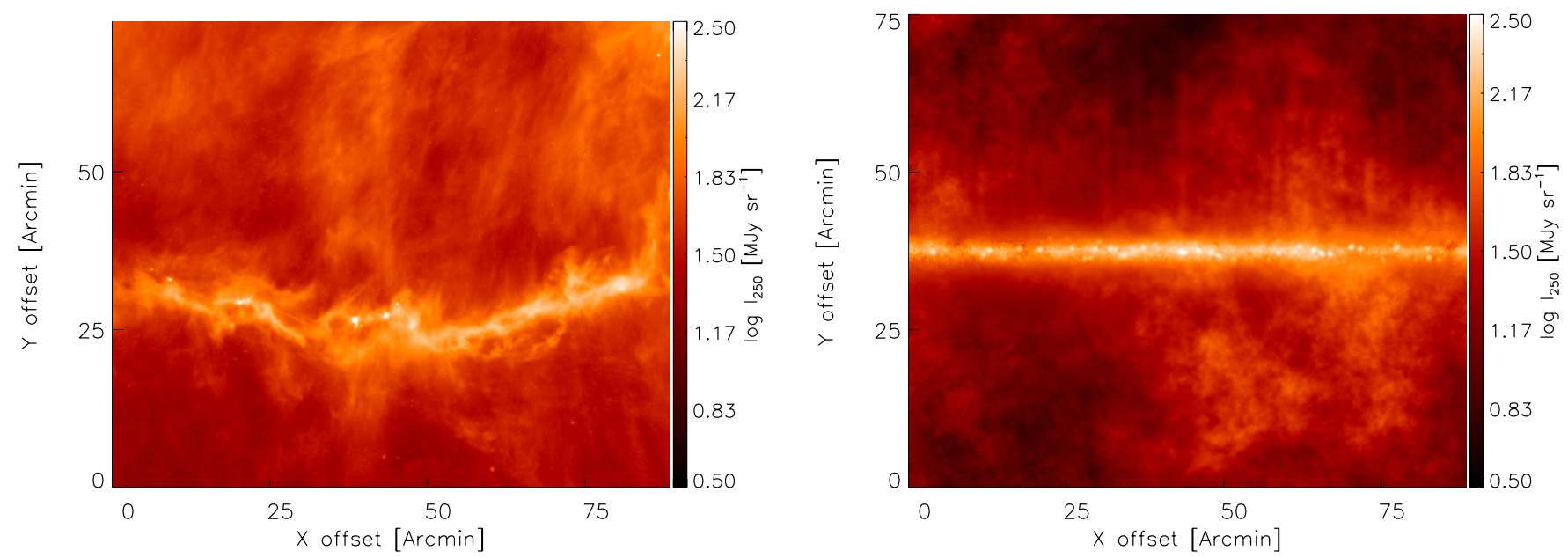

Fig. 9. Left panel: Herschel/SPIRE $250 \mu \mathrm{m}$ image of the B211/B213 region in Taurus at the native beam resolution of $18.2^{\prime \prime}$, but rotated in equatorial coordinates in clockwise direction by $37.4^{\circ}$. (see Palmeirim et al. 2013). Right panel: fully synthetic image mimicking the main features of the real image shown in the left panel, and resulting from the co-addition of a synthetic filament image and a synthetic background image. The synthetic filament image was based on the Plummer-like model of the B211 filament reported by Palmeirim et al. (2013): flat inner radius $R_{\text {flat }}=0.035 \mathrm{pc}$, contrast $\delta_{\mathrm{c}} \sim 6$, and power-law index $p=2$ at large radii. The background image was modeled as non-Gaussian cirrus fluctuations with a logarithmic power spectrum slope of -3 (see text for details), plus low-contrast filamentary structures resembling striations. The synthetic striations were placed such that their long axis is perpendicular to the main filament at a regular separation of $0.1 \mathrm{pc}$.

\section{Power spectrum of synthetic data with a single, prominent filament}

We also examined the power spectrum of an image with a single dominant filament such as the Herschel/SPIRE $250 \mu \mathrm{m}$ image of the B211/B213 region in the Taurus cloud at $d \sim 140 \mathrm{pc}$ (Fig. 9a $)^{8}$. For the present purpose, we only used a $1.2^{\circ} \times 1.0^{\circ}$ portion of the original SPIRE image of B211/B213, where a single filament dominates over a length scale of $>1.5^{\circ}$ (or $>4 \mathrm{pc}$ ). Palmeirim et al. (2013) studied the column density structure of the B211/B213 filament in detail and found that it is accurately described by a Plummer-like cylindrical density distribution with flat inner radius $R_{\text {flat }} \sim 0.035 \mathrm{pc}$ and power-law index $p=2 \pm 0.2$ at larger radii up to an outer radius $R_{\text {out }} \sim 0.4 \mathrm{pc}$. Moreover, Palmeirim et al. (2013) suggested that the Taurus main filament accretes mass from the ambient cloud through a network of lower-density striations, observed roughly perpendicular to the main filament. Based on these findings, we constructed a synthetic image of a Plummer-like filament of length $\sim 4 \mathrm{pc}$, with the same Plummer parameters as quoted above, and positioned horizontally in a $\sim 1.5^{\circ} \times 1.5^{\circ}$ two-dimensional box. The contrast of the synthetic filament was chosen to be $\delta_{\mathrm{c}} \sim 6$, a value close to the observed contrast of the B211/B213 filament in the SPIRE $250 \mu \mathrm{m}$ image (see Palmeirim et al. 2013). To mimic the observations, we added a population of synthetic cores with Bonnor-Ebert-like radial profiles randomly distributed along the filament. The flat inner radius $R_{\text {flat }}$ of the cores was fixed to a constant value of $0.02 \mathrm{pc}$. In order to create a synthetic background image similar to the real data, we carefully studied the statistical properties of the Herschel $250 \mu \mathrm{m}$ image in the vicinity of the Taurus main filament. We selected a rectangular field to the north of the B211/B213 main filament such that the nearest edge of the field was at least $0.2 \mathrm{pc}$ away from the filament crest. We then evaluated the power spectrum of this field and found a logarithmic slope $\gamma \sim-3.0 \pm 0.2$. A purely synthetic background image was next generated using a non-Gaussian fractional Brow-

\footnotetext{
8 In order to capture the largest possible rectangular area, we rotated the SPIRE map by $37.4^{\circ}$ in the clockwise direction with respect to an equatorial frame.
}

nian motion (fBm) technique (Miville-Deschênes et al. 2003) with positive values and statistics such that the power spectrum of the background field had a logarithmic slope similar to that of the Taurus background field $\left(\gamma_{\text {back }}=-3.0\right)$. To make the synthetic background image more similar to the Taurus observations, we also inserted a distribution of lognormally distributed low-contrast $\left(0.1<\delta_{\mathrm{c}}<0.5\right)$ filamentary structures with Gaussian profiles perpendicular to the main filament as a proxy for the observed striations. We placed perpendicular striations at a regular separation of $\sim 0.1 \mathrm{pc}$ to match the observations of Tritsis \& Tassis (2018). The width of these synthetic striations was fixed to $0.08 \mathrm{pc}$

The final image, obtained after co-adding all three synthetic image components (background, striations, and main filament with embedded cores), is shown in Fig. 9b. For reference and comparison with the synthetic data discussed in Sects. 4 and 5 , this image has $\delta_{\mathrm{c}}^{2} A_{\mathrm{fil}} \sim 0.125$. Figure 10 compares the power spectrum of the synthetic image (red curve) with that of the Herschel/SPIRE $250 \mu \mathrm{m}$ image (black solid curve). The vertical dashed line in Fig. 10 marks the angular frequency $k_{\text {fil }}$ corresponding to a linear scale of $\sim 0.1 \mathrm{pc}$, i.e., roughly the inner width of both the synthetic filament and the B211/B213 filament. Clearly, like for the other two regions considered in this paper, the power spectrum of the Taurus B211/B213 data does not reveal any "kink" or "break" at frequencies close to $k_{\text {fil }}$. Furthermore, this is also the case for the synthetic data of Fig. 9b, despite the presence of a prominent cylindrical filament with $\sim 0.1 \mathrm{pc}$ inner diameter. This further illustrates how the characteristic scale of embedded structures may be hidden and undetectable in a global power spectrum.

\section{Combined effect of filament contrast and area filling factor}

In order to further explore the dependence of the total power spectrum on filament contrast and area filling factor, we performed two separate grids of $20 \times 20$ Monte-Carlo simulations based on two different sets of synthetic filament populations, one with Gaussian radial profiles and the other with Plummer-like profiles with $p=2$ (see Appendix A). The simulated images spanned a 


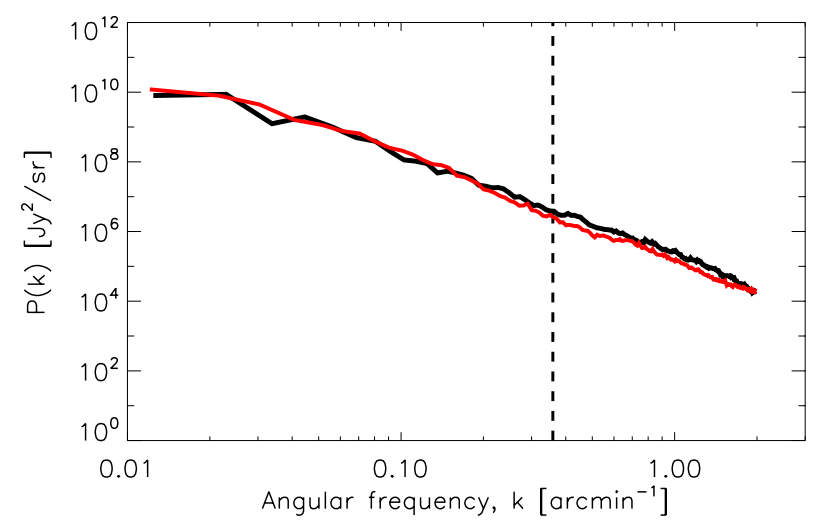

Fig. 10. Comparison of the power spectrum of the Herschel/SPIRE $250 \mu \mathrm{m}$ image of Fig. 9a (black solid curve) with that of the synthetic image of Fig. 9b (red curve). Note the absence of any significant feature around $k \sim k_{\text {fil }}$ (vertical dashed line) in both power spectra.

broad range of average filament contrasts $\left\langle\delta_{\mathrm{c}}\right\rangle$ and filling factors $A_{\text {fil }}$. In practice, we injected a fixed number of synthetic filaments of $0.1 \mathrm{pc}$ width in the Herschel/SPIRE $250 \mu \mathrm{m}$ image of Polaris and controlled the area filling factor by varying the length of the filaments. For each realization, we then calculated the $\chi_{\text {variance }}^{2}$ of the residuals between the best power-law fit and the net output power spectrum, as described in Sect. 3.

Figure 11 summarizes the dependence of $\chi_{\text {variance }}^{2}$ on $\left\langle\delta_{\mathrm{c}}\right\rangle^{2}$ and $A_{\text {fil }}$ for Gaussian synthetic filaments. The map of the $\chi_{\text {variance }}^{2}$ as a function of $\left\langle\delta_{\mathrm{c}}\right\rangle^{2}$ and $A_{\text {fil }}$ is qualitatively similar for Plummer-like synthetic filaments. Figure 12 shows that there is a tight correlation between $\chi_{\text {variance }}^{2}$ and $\left\langle\delta_{\mathrm{c}}\right\rangle^{2} \times A_{\text {fil }}$ for both Gaussian (black solid circles) and Plummer-like filaments (gray solid squares), as expected from Eq. (12). In both cases, $\chi_{\text {variance }}^{2}$ appears to be a non-linear function of $\delta_{\mathrm{c}}^{2} A_{\mathrm{fil}}$, with a flat portion at low $\delta_{\mathrm{c}}^{2} A_{\text {fil }}$ values (i.e., $\delta_{\mathrm{c}}^{2} A_{\text {fil }} \lesssim 0.02$ for Gaussian filaments, $\delta_{\mathrm{c}}^{2} A_{\mathrm{fil}} \lesssim$ 0.07 for Plummer-like filaments), a rising portion at higher $\delta_{\mathrm{c}}^{2} A_{\text {fil }}$ values, with an inflection point close to $\delta_{\mathrm{c}}^{2} A_{\mathrm{fil}} \sim 0.1$ in the Gaussian case and $\delta_{\mathrm{c}}^{2} A_{\text {fil }} \sim 0.4$ in the Plummer case (see Fig. 12). It can also be seen that, for the same value of $\delta_{\mathrm{c}}^{2} A_{\mathrm{fil}}$, the $\chi_{\text {variance }}^{2}$ is lower for Plummer synthetic filaments than for Gaussian synthetic filaments ${ }^{9}$.

Qualitatively, this behavior may be understood as follows. At low $\delta_{\mathrm{c}}^{2} A_{\text {fil }} \lesssim 0.02$ values (or $\delta_{\mathrm{c}}^{2} A_{\text {fil }} \lesssim 0.07$ for Plummer-like filaments), the contribution of synthetic filaments to the total power spectrum is negligible, and $\chi_{\text {Variance }}^{2}$ is dominated solely by the residuals of the original background image. Therefore, $\chi_{\text {variance }}^{2}$ retains the value $\chi_{\text {variance,bkg }}^{2}$ it has for the original image and remains constant despite the addition of synthetic filaments. As $\delta_{\mathrm{c}}^{2} A_{\mathrm{fil}}$ increases, the $\chi_{\text {variance }}^{2}$ for both Gaussian and Plummer filaments also increases, reaching a value of about $3 \times \chi_{\text {variance,bkg }}^{2} \sim 0.1$ at $\delta_{\mathrm{c}}^{2} A_{\text {fil }} \sim 0.1$ (Gaussian case) or 0.4 (Plummer case). We take these values of $\delta_{\mathrm{c}}^{2} A_{\text {fil }}$ as fiducial limits for the detection of a characteristic filament width in the image power spectrum for Gaussian- and Plummer-shaped filaments, respectively. These fiducial detection limits are marked by black and gray vertical dashed lines in Fig. 12 .

To put the simulation results shown in Figs. 11 and 12 in context, we recall that the Polaris simulation of Fig. 5a in Sect. 3 had

\footnotetext{
9 A Plummer-like filament with $p \lesssim 2.6$ contributes less power to the power spectrum at low angular frequency than a Gaussian filament with similar inner width and contrast (see Fig. 2b). Therefore, at $k<k_{\text {fil }}$, Plummer-like filaments with $p \lesssim 2.6$ lead to a lower overall $\chi_{\text {Variance }}^{2}$ compared to Gaussian filaments.
}

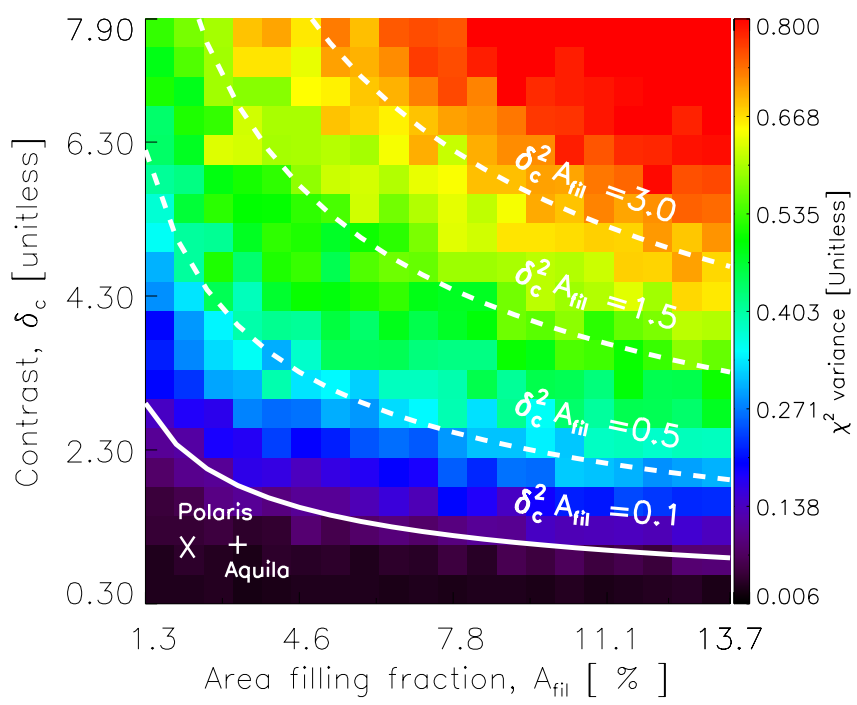

Fig. 11. Map of the $\chi^{2}-$ variance of the residuals between the best power-law fit and the output power spectrum as a function of column density contrast $\left(\delta_{\mathrm{c}}\right)$ and area filling factor $\left(A_{\mathrm{fil}}\right)$ in a grid of simulations based on a set of $20 \times 20$ populations of Gaussian synthetic filaments, all $0.1 \mathrm{pc}$ in width (see text of Sect. 7 for details). The white solid curve marks the fiducial limit $\delta_{\mathrm{c}}^{2} A_{\mathrm{fil}}=0.1$ above which the effect of the characteristic filament width can be detected in the power spectrum (see Fig. 12 below). The white plus and cross symbols mark the positions of the observed populations of filaments in the Aquila and Polaris clouds, respectively (cf. Arzoumanian et al. 2019).

$\delta_{\mathrm{c}}^{2} A_{\mathrm{fil}} \sim 0.018$ and $\chi_{\text {variance }}^{2}$ of 0.034 (see Fig. $4 \mathrm{c}$ ), which is nearly the same as the observed $\chi_{\text {variance,bkg }}^{2} \sim 0.04$ (see Fig. 3). This particular simulation is marked by a blue triangle in the $\chi_{\text {variance }}^{2}$ $\delta_{\mathrm{c}}^{2} A_{\text {fil }}$ plot of Fig. 12. The more extreme Polaris simulation presented in Fig. B.2a, for which there is a marginal detection of a characteristic scale in the residuals plot (see Fig. B.2c), has $\delta_{\mathrm{c}}^{2} A_{\text {fil }} \sim 0.087$ and $\chi_{\text {variance }}^{2} \sim 0.08$ (see red triangle in Fig. 12). Likewise, the blue and red square symbols in the $\chi_{\text {variance }}^{2}-\delta_{\mathrm{c}}^{2} A_{\text {fil }}$ plot of Fig. 12 mark the positions of the two sets of Aquila simulations presented in Figs. 8 and B.3, respectively.

The red square in Fig. 12 has $\delta_{\mathrm{c}}^{2} A_{\text {fil }} \sim 0.27$ (and $\chi_{\text {variance }} \sim$ 0.28 ), significantly above the fiducial detection limit of 0.1 , indicating that the signature of a characteristic filament width should be detectable in the power spectrum. This is indeed confirmed by visual inspection of Fig. B.3b and c. Most importantly, for both Polaris and Aquila, the real Herschel data lie in a portion of the $\chi_{\text {variance }}^{2}-\delta_{\mathrm{c}}^{2} A_{\mathrm{fil}}$ diagram where the filament contribution has a negligible impact on the power spectrum (see cross and plus symbols in Figs. 11 and 12). Also shown as a green filled circle in Fig. 12 is the locus of the Herschel data for the prominent filament system B211/B213 in Taurus (see Sect. 6), which has a very well characterized Plummer-like density profile with a power-law wing index $p=2 \pm 0.2$ (Palmeirim et al. 2013). It can be seen that the position of the Taurus B211/B213 data in Fig. 12 is in excellent agreement with our set of simulations for Plummer-shaped filaments with $p=2$. Although the $\delta_{\mathrm{c}}^{2} A_{\mathrm{fil}} \sim 0.125$ value of the Taurus B211/B213 data is greater than the fiducial threshold for Gaussian filaments, it remains much lower than the fiducial detection limit for Plummer $(p=2)$ filaments.

We conclude that the essentially scale-free power spectrum of the Herschel images observed toward molecular clouds such as Polaris, Aquila, or Taurus does not invalidate the existence of a characteristic filament width. 


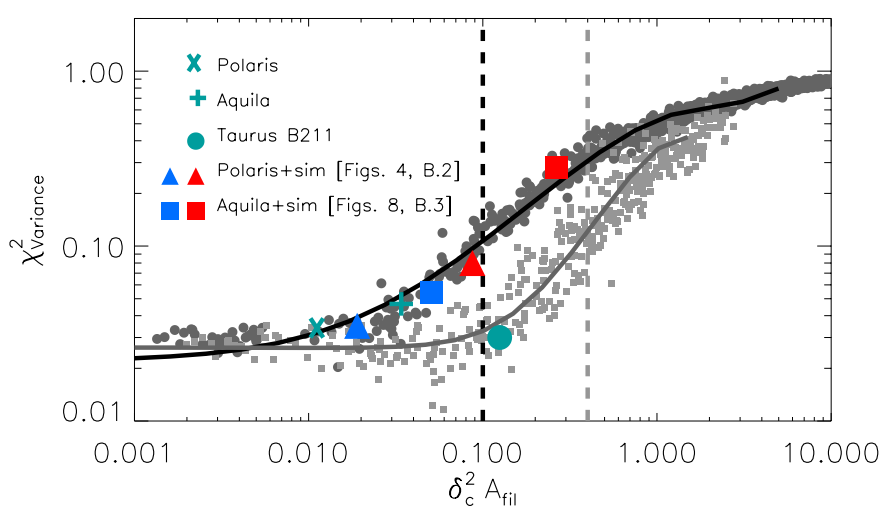

Fig. 12. $\chi_{\text {Variance }}^{2}$ of the residuals between the best power-law fit and the output power spectrum as a function of $\delta_{\mathrm{c}}^{2} A_{\mathrm{fil}}$. The black solid circles represent the same set of simulations with Gaussian filaments as in Fig. 11. The light gray squares represent our set of simulations with Plummerlike $(p=2)$ filament profiles. The corresponding black and gray curves are polynomial fits to guide the eye. The black and gray vertical dashed lines mark the fiducial limits of $\delta_{\mathrm{c}}^{2} A_{\text {fil }} \sim 0.1$ and $\delta_{\mathrm{c}}^{2} A_{\text {fil }} \sim 0.4$ above which Gaussian-shaped and Plummer-shaped filaments become detectable in the residual power spectrum plot, respectively. The green cross, plus, and solid circle symbols mark the positions of the Polaris, Aquila, and Taurus clouds, respectively, based on the comprehensive study of filament properties by Arzoumanian et al. (2019).

\section{Summary and conclusions}

We used numerical experiments to investigate the conditions under which the presence of a characteristic filament width can manifest itself in the power spectrum of cloud images. Our main findings and conclusions may be summarized as follows:

1. The detectability of a characteristic filament scale in the power spectrum of an ISM dust continuum image primarily depends on the parameter $\delta_{\mathrm{c}}^{2} A_{\mathrm{fil}}$, where $\delta_{\mathrm{c}}$ is the weighted average column density contrast of the filamentary structures and $A_{\text {fil }}$ their area filling factor in the image. A value $\delta_{\mathrm{c}}^{2} A_{\mathrm{fil}} \gtrsim 0.1$ is required for the presence of a characteristic filament width to produce a significant signature in the power spectrum.

2. The Herschel Gould Belt survey images of nearby clouds typically have $\delta_{\mathrm{c}}^{2} A_{\mathrm{fil}} \ll 0.1$ and therefore lie in a region of the parameter space where filaments have a negligible impact on the power spectrum. Therefore, despite recent claims, the scale-free nature of the observed power spectra remains consistent with the presence of a characteristic filament width $\sim 0.1 \mathrm{pc}$.

3. When the average filament contrast is low and/or when the filaments occupy a small area filling factor, the power spectrum is dominated by the fluctuations of the diffuse, nonfilamentary component of the ISM.

4. Although a few filaments in the Polaris cloud have column density contrasts up to $\delta_{\mathrm{c}} \sim 0.9$, their area filling factor is extremely low $A_{\text {fil }} \sim 2 \%$, resulting in a combined parameter $\delta_{\mathrm{c}}^{2} A_{\mathrm{fil}} \sim 0.01$ for Polaris. The overall power spectrum of the Herschel images of Polaris is scale-free because the filaments are not contributing enough power to produce a significant signature at the spatial frequency corresponding to the characteristic filament width of $\sim 0.1 \mathrm{pc}$.

5. Despite the presence of several supercritical filaments of $\sim 0.1 \mathrm{pc}$ inner width in the Aquila cloud, the power spectrum of the Aquila Herschel images is also essentially scale free. Due to the larger distance of the Aquila cloud compared to Polaris, $\sim 0.1 \mathrm{pc}$ filaments in Aquila subtend a smaller angu- lar width scale on the sky, and therefore have a relatively low area filling factor. Overall, our simulations suggest that the observed population of Aquila filaments contributes only $\sim 1 / 5$ of the total amplitude of the power spectrum of the Herschel $250 \mu \mathrm{m}$ image.

6. Supercritical filaments with Plummer-like profiles and high column density contrasts lead to relatively small departures from a power-law power spectrum because the high contrast of the flat inner plateau in the density profile is compensated by broad power-law wings at large radii. The B211/B213 filament system in Taurus, for example, despite having a very high central column density contrast, remains largely undetected in the image power spectrum because of its Plummerlike density profile with $p \approx 2$.

7. We conclude that the scale-free appearance of the power spectra of cloud images does not invalidate the finding, based on detailed Herschel studies of the column density profiles, that nearby molecular filaments have a common inner width $\sim 0.1$ pc (Arzoumanian et al. 2011, 2019).

Acknowledgements. This work has received support from the European Research Council under the European Union's Seventh Framework Programme (ERC Advanced Grant Agreement no. 291294 - "ORISTARS”). We also acknowledge financial support from the French national programs of CNRS/INSU on stellar and ISM physics (PNPS and PCMI). A.R, and N.S., acknowledge support by the French ANR and the German DFG through the project "GENESIS" (ANR-16-CE92-0035-01/DFG1591/2-1). P.P. acknowledges support from the Fundação para a Ciência e a Tecnologia of Portugal (FCT) through national funds (UID/FIS/04434/2013), from FEDER through COMPETE2020 (POCI-01-0145-FEDER-007672), and from the fellowship SFRH/BPD/110176/2015 funded by FCT (Portugal) and POPH/FSE (EC). We are grateful to our colleague Alexander Men'shchikov for assistance with the getfilaments algorithm. This research has made use of data from the Herschel Gould Belt survey (HGBS) project (http://gouldbelt-herschel.cea.fr). The HGBS is a Herschel Key Programme jointly carried out by SPIRE Specialist Astronomy Group 3 (SAG 3), scientists of several institutes in the PACS Consortium (CEA Saclay, INAF-IFSI Rome and INAF-Arcetri, KU Leuven, MPIA Heidelberg), and scientists of the Herschel Science Center (HSC).

\section{References}

André, P., Men'shchikov, A., Bontemps, S., et al. 2010, A\&A, 518, L102 André, P., Di Francesco, J., Ward-Thompson, D., et al. 2014, Protostars and Planets VI, 27

Arzoumanian, D., André, P., Didelon, P., et al. 2011, A\&A, 529, L6

Arzoumanian, D., André, P., Könyves, V., et al. 2019, A\&A, 621, A42

Auddy, S., Basu, S., \& Kudoh, T. 2016, ApJ, 831, 46

Falgarone, E., Panis, J.-F., Heithausen, A., et al. 1998, A\&A, 331, 669

Federrath, C. 2016, MNRAS, 457, 375

Fischera, J., \& Martin, P. G. 2012, A\&A, 542, A77

Gao, Y., \& Solomon, P. M. 2004, ApJ, 606, 271

Heiderman, A., Evans, II., N. J., Allen, L. E., Huard, T., \& Heyer, M. 2010, ApJ, 723, 1019

Hennebelle, P., \& André, P. 2013, A\&A, 560, A68

Inutsuka, S., \& Miyama, S. M. 1997, ApJ, 480, 681

Koch, E. W., \& Rosolowsky, E. W. 2015, MNRAS, 452, 3435

Könyves, V., André, P., Men'shchikov, A., et al. 2015, A\&A, 584, A91

Lada, C. J., Lombardi, M., \& Alves, J. F. 2010, ApJ, 724, 687

Marsh, K. A., Kirk, J. M., André, P., et al. 2016, MNRAS, 459, 342

Martin, P. G., Miville-Deschênes, M.-A., Roy, A., et al. 2010, A\&A, 518, L105

Men'shchikov, A. 2013, A\&A, 560, A63

Miville-Deschênes, M.-A., Levrier, F., \& Falgarone, E. 2003, ApJ, 593, 831

Miville-Deschênes, M.-A., Martin, P. G., Abergel, A., et al. 2010, A\&A, 518, L104

Palmeirim, P., André, P., Kirk, J., et al. 2013, A\&A, 550, A38

Panopoulou, G. V., Psaradaki, I., Skalidis, R., Tassis, K., \& Andrews, J. J. 2017, MNRAS, 466, 2529

Roy, A., André, P., Arzoumanian, D., et al. 2015, A\&A, 584, A111

Schneider, N., André, P., Könyves, V., et al. 2013, ApJ, 766, L17

Shimajiri, Y., André, P., Braine, J., et al. 2017, A\&A, 604, A74

Tritsis, A., \& Tassis, K. 2018, Science, 360, 635

Ward-Thompson, D., Kirk, J. M., André, P., et al. 2010, A\&A, 518, L92 


\section{Appendix A: Construction of synthetic filaments with Plummer-like density profiles}

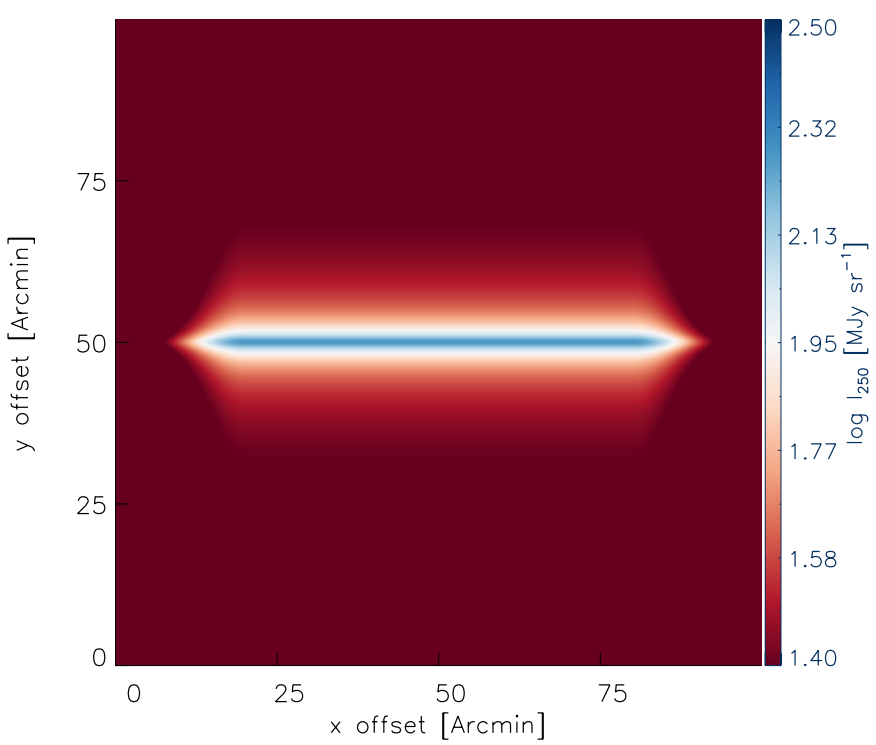

Fig. A.1. Image of a synthetic filament with a Plummer-like transverse density profile with a flat inner radius, $R_{\text {flat }}=0.03 \mathrm{pc}$, and a powerlaw wing with index $p=2$, projected at a distance of $140 \mathrm{pc}$. In this example, the level of filament contrast was adjusted to $\delta_{\mathrm{c}} \sim 10$.

We adopted a slightly different technique to produce filaments with Plummer profiles compared to the convolution technique used to generate filaments with Gaussian profiles (see Sect. 2). A Plummer-like transverse profile was first constructed using the expression

$K_{\text {Plummer }}(r)=\frac{C}{\left[1+\left(r / R_{\text {flat }}\right)^{2}\right]^{(p-1) / 2}}$,

where $R_{\text {flat }}$ is the flat inner width and $p$ is the logarithmic slope of the power-law wing at large radii $\left(r>>R_{\text {flat }}\right)$. In order to suppress the strong edge effect at the two ends of the model filament, we tapered both edges with a Gaussian function. Figure A.1 shows an example of synthetic filament with a Plummer profile $p=2$ and $R_{\text {flat }}=0.1 \mathrm{pc}$, similar to the Taurus B211 filament (Palmeirim et al. 2013). The power spectra of synthetic filaments with Plummer-like density profiles are discussed in Sect. 2.

\section{Appendix B: Effect of extreme filament contrasts and area filling factors on the power spectrum}

Figures B.2 and B.3 illustrate the consequences of adding populations of synthetic filaments with very high column density contrasts on the total power spectra of Polaris and Aquila, respectively. Figure B.2a displays the Herschel $250 \mu \mathrm{m}$ image of the Polaris cloud populated with a set of high-contrast filaments with $\delta_{\mathrm{c}} \sim 1.1$. The number of synthetic filaments was fixed to 100 and the distribution of synthetic filament lengths was adjusted so that the overall area filling factor was around $A_{\text {fil }} \sim 7 \%$. In this case, the synthetic filaments contribute a level of power (blue curve) almost equivalent to the power spectrum of the Polaris original image (cf. dashed green curve). This leads to an enhancement of power in the total power spectrum, which can be clearly seen in the residuals plot (red filled circles in Fig. B.2c). The $\chi^{2}-$ variance of the residuals of the power-law
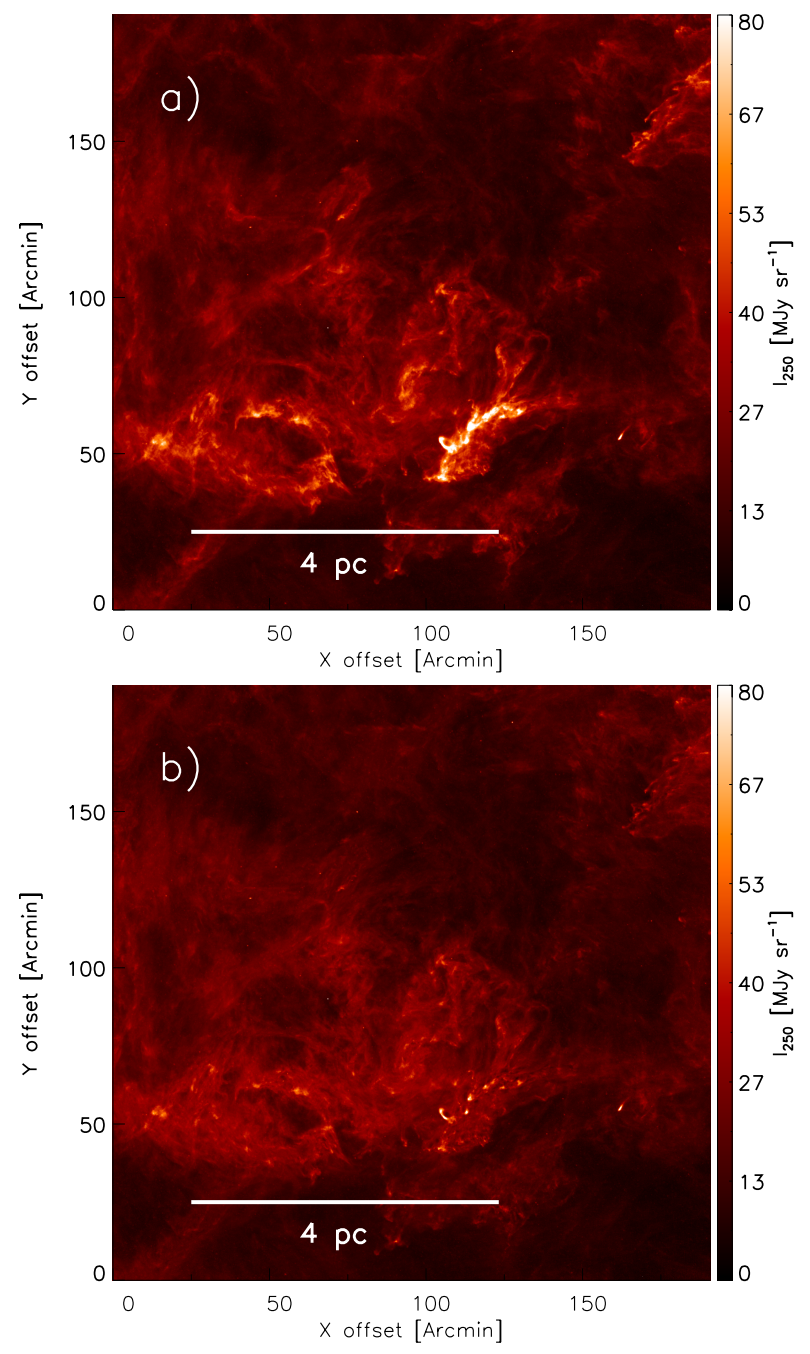

Fig. B.1. Comparison of the Herschel/SPIRE $250 \mu \mathrm{m}$ image of Polaris at the native beam resolution of 18.2" (panel a-see Miville-Deschênes et al. 2010) with the filament-subtracted image of the same field, panel $b$ obtained with the getfilaments algorithm (Men'shchikov 2013) and used as a "filament-free" background image in the numerical experiments shown in Figs. 5 and B.2.

fit in the angular frequency range of $k_{\min }<k<1.5 k_{\mathrm{fil}}$ is about seven times larger than the $\chi^{2}-$ variance metric for the Polaris original image.

In the Aquila case, we created a population of 100 synthetic filaments rescaling the observed distribution of column density contrasts as shown in Fig. B.4. The maximum contrast sampled in the distribution was increased to $\delta_{\mathrm{c}}^{\max }=15$ (compared to $\sim 3$ in the original contrast distribution), and the peak of the distribution was shifted to $\delta_{\mathrm{c}}^{\text {peak }} \sim 2$ compared to 1 in the original distribution. The average contrast level of the synthetic filaments was about 2.2 and their area filling factor was as high as $5.5 \%$. The resulting image obtained after adding this population of synthetic filaments to the Aquila original image is shown in Fig. B.3a. The power contribution due to the synthetic filaments, shown by the blue curve in Fig. B.3b, is significantly higher than the power spectrum amplitude of the Aquila original image (dashed green curve). Accordingly, the total power spectrum, $\left[P_{\text {fil }}(k)+P_{\text {Aquila }}(k)\right.$, solid black curve in Fig. B.3b] is amplified at angular frequencies $k \lesssim k_{\text {fil }}$. A strong deviation in the residuals plot (red symbols in Fig. B.3c) can also be seen. 

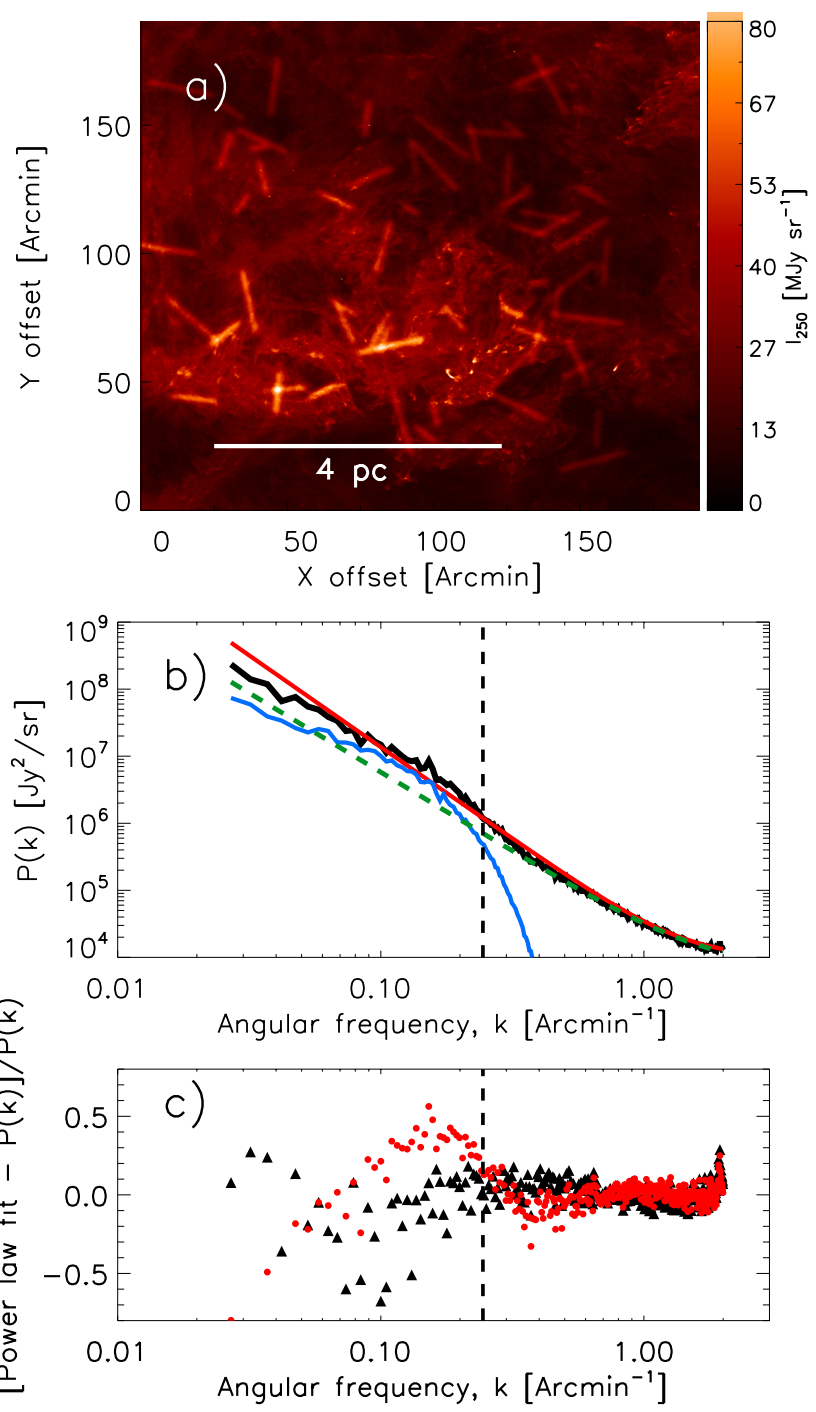

Fig. B.2. Same as Fig. 5 but for a population of synthetic filaments with higher column density contrasts $\delta_{\mathrm{c}} \sim 1.1$, resulting in $\delta_{\mathrm{c}}^{2} A_{\mathrm{fil}} \sim 0.087$. (The area filling factor is similar to that in Fig. $4, A_{\mathrm{fil}} \sim 7.2 \%$.) Panel $b$ : note how the amplitude of the power spectrum due to the synthetic filaments (blue curve) is comparable to that of the power spectrum of the Polaris original image (see green dashed line and Fig. 3). The logarithmic slope of the total power spectrum $P(k)_{\text {fil }}+P(k)_{\text {Polaris }}$ is -2.96 Panel $c$ : residuals between the best power-law fit and $P(k)_{\text {Polaris }+ \text { fil }}$ (red solid circles) shows a peak near $k_{\text {fil }} \sim 0.24 \operatorname{arcmin}^{-1}$. In this simulation, the $\chi_{\text {variance }}^{2}$ is 0.08 , close to the fiducial detection limit $\delta_{\mathrm{c}}^{2} A_{\text {fil }} \sim 1$ introduced in Sect. 7 (see Fig. 12).
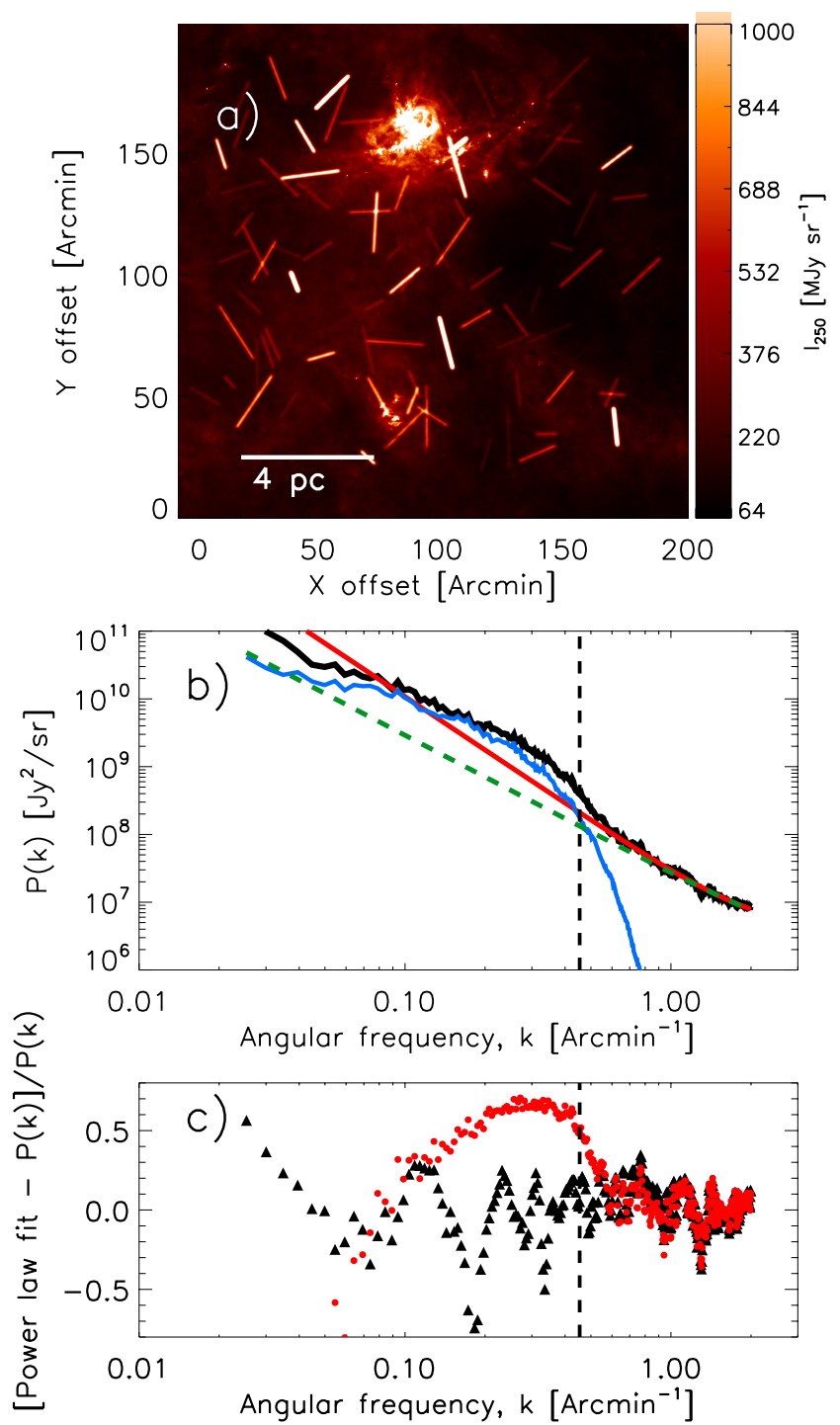

Fig. B.3. Same as Fig. 8 but for a population of synthetic filaments with a more extreme (and unrealistic) distribution of column density contrasts corresponding to $\left\langle\delta_{\mathrm{c}}\right\rangle \sim 2.2$ (see Fig. B.4) and an area filling factor $A_{\text {fil }} \sim 5.5 \%$, resulting in a combined parameter $\delta_{\mathrm{c}}^{2} A_{\mathrm{fil}} \sim 0.27$. Panel $b$ : note how the power spectrum arising from the synthetic filament population (blue curve) dominates over the power spectrum of the Aquila original image (see green dashed curve and Fig. 6). The logarithmic slope of the best power-law fit to the total power spectrum $P(k)_{\text {fil }}+P(k)_{\text {Aquila }}$ is -2.6 (red line). Panel $c$ : residuals between the best power-law fit and $P(k)_{\text {Aquilatfil }}$ (red solid circles) shows a peak near $k_{\text {fil,Aquila }}=0.45 \mathrm{arcmin}^{-1}$. For this simulation, the $\chi_{\text {Variance }}^{2}$ of the residuals is 0.28 . 


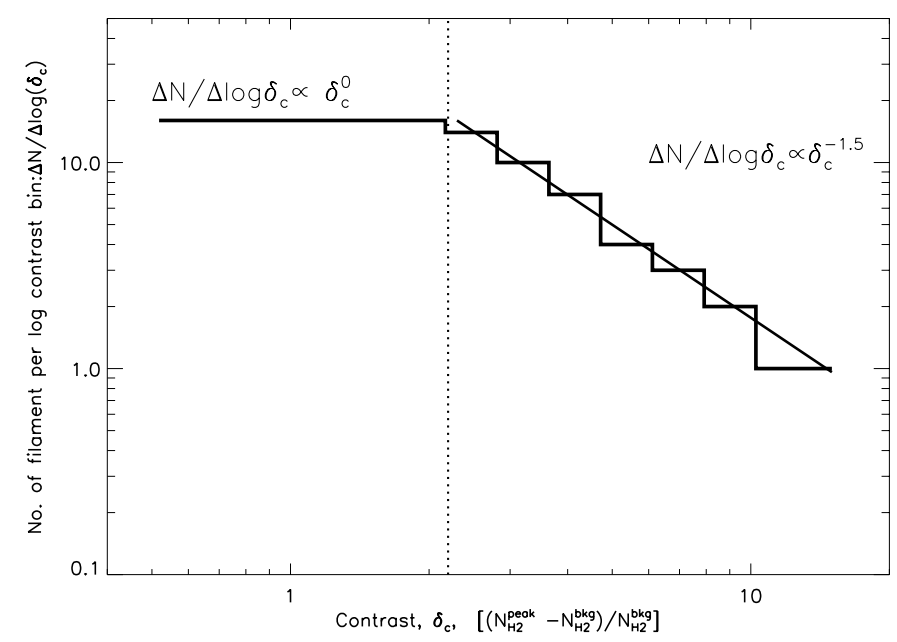

Fig. B.4. Two-segment power-law distribution of synthetic filaments contrasts adopted in the Aquila simulations of Appendix B. The distribution ranges from 0.3 to 15 and leads to a weighted average contrast of $\sim 2.2$, significantly higher than in Fig. 7 .

\section{Appendix C: Effect of filaments embedded in a scale-free synthetic background}

We also generated a purely synthetic background image using the non-Gaussian fractional Brownian motion ( $\mathrm{fBm}$ ) technique of Miville-Deschênes et al. (2003), in such a way that the resulting power spectrum had a logarithmic slope $\gamma=-2.7$, mean brightness of the fluctuation $\sim 17 \mathrm{MJy} / \mathrm{sr}$, and a standard deviation of $10 \mathrm{MJy} / \mathrm{sr}$, similar to the statistics observed with Herschel in the Polaris field. On top of this scale-free image, a population of synthetic filaments with a lognormal distribution of $\left(\delta_{\mathrm{c}}\right)$ contrasts (similar to that observed in Polaris) was added. The filaments had a Gaussian profile with an inner width of $0.1 \mathrm{pc}$ projected at a distance of $140 \mathrm{pc}$, similar to the distance of the Polaris molecular cloud. They occupied an area-filling factor $A_{\text {fil }} \sim 3 \%$ and had an average contrast $\delta_{\mathrm{c}} \sim 0.8$. The resulting synthetic image after co-adding the $\mathrm{fBm}$ background image and the synthetic filaments is shown in Fig. C.1a. Inspection of the various components of the image power spectrum (shown in Fig. C.1b) shows that the contribution of the synthetic filaments to the global power spectrum is negligible and undetectable in this case as well.
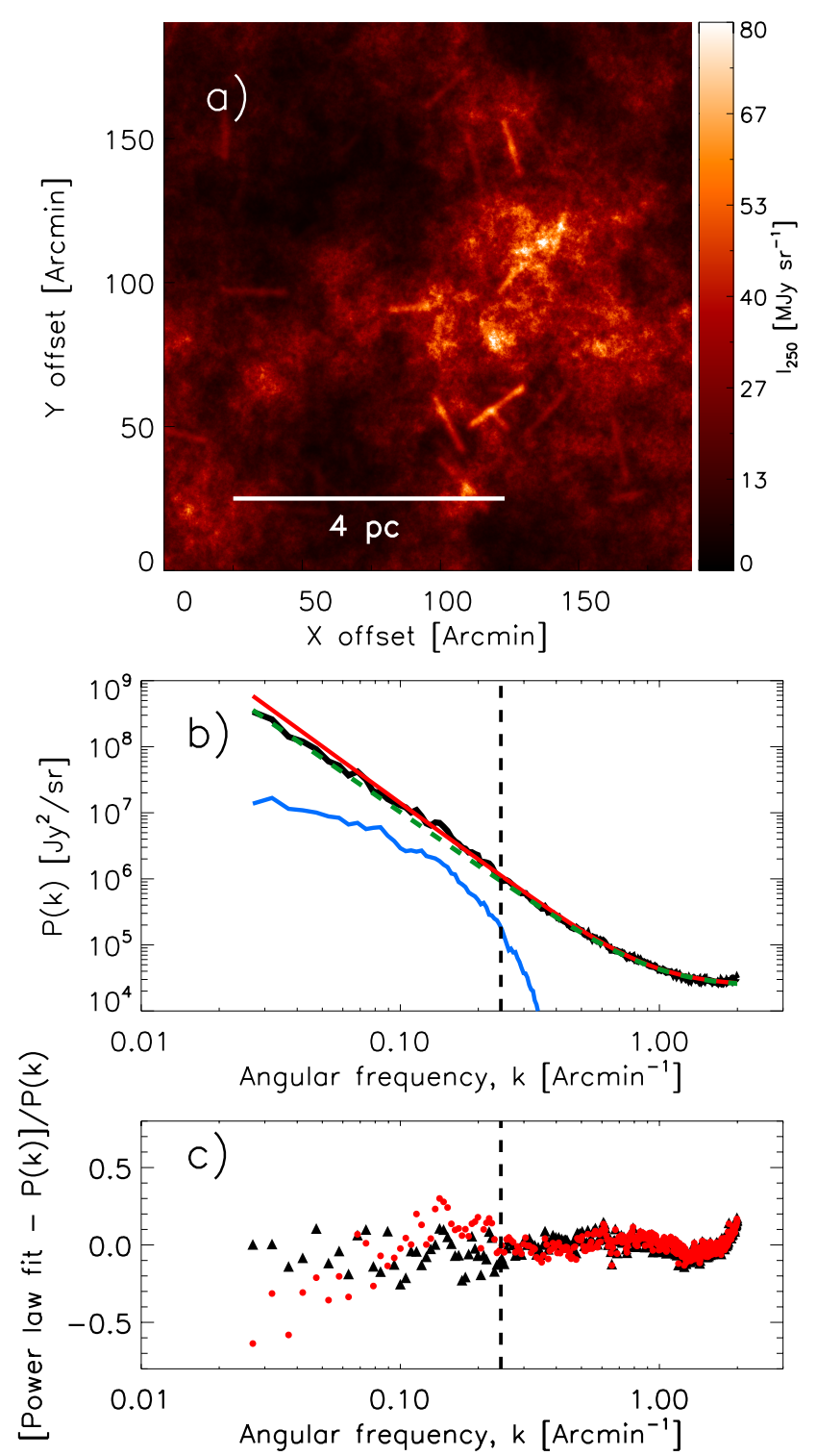

Fig. C.1. Purely synthetic image, made up of a scale-free background image constructed using the $\mathrm{fBm}$ algorithm (panel $a$; cf. Miville-Deschênes et al. 2003). The embedded synthetic filaments have a lognormal column density contrast distribution in a range between $0.3<\delta_{\mathrm{c}}<2$ and peak of $\delta_{\text {peak }} \sim 0.9$. The overall area-filling factor of the filaments is $A_{\mathrm{fil}} \sim 3 \%$. The filaments are of Gaussian profiles with a FWHM $\sim 0.1 \mathrm{pc}$ at a distance of $140 \mathrm{pc}$ (see text). Panel $b$ : solid black curve shows the power spectrum of the scale-free background image and the synthetic filaments. The logarithmic slope of the power-spectrum is $\gamma \sim-2.7 \pm 0.1$. The dashed curve shows the power spectrum of the background image $(\gamma \sim-2.8)$. The blue solid line shows the power spectrum of the synthetic filaments. Panel $c$ : residuals between the best power-law fit and the power spectrum data points (triangle symbols) of synthetic cirrus map. The $\chi_{\text {Variance }}^{2}$ of the residuals between $k_{\min }<k<1.5 k_{\text {fil }}$ is 0.03 . 